\title{
Identifiability of Rank-3 Tensors
}

\author{
Edoardo Ballico, Alessandra Bernardi and Pierpaola Santarsiero®
}

\begin{abstract}
Rank-2 and rank-3 tensors are almost all identifiable with only few exceptions.We classify them all together with the dimensions and the structures of all the sets evincing the rank.
\end{abstract}

Mathematics Subject Classification. 14N07, 15A69.

\section{Introduction}

Identifiability of tensors is one of the most active research areas both in pure mathematics and in applications. The core of the problem is being able to understand if a given tensor $T \in \mathbb{C}^{n_{1}+1} \otimes \cdots \otimes \mathbb{C}^{n_{k}+1}$ can be decomposed in a unique way as a sum of pure tensors:

$$
T=\sum_{i=1}^{r} v_{1, i} \otimes \cdots \otimes v_{k, i}
$$

with $v_{j, i} \in \mathbb{C}^{n_{j}+1}$, for $j=1, \ldots, k$. Of course the minimum $r$ realizing the above expression is a crucial value and it is called the rank of $T$.

From the applied point of view, identifiability in tensor decomposition arises naturally in numerous areas, we quote as examples Phylogenetics, Quantum Physics, Complexity Theory and Signal Processing (cf. e.g. $[3,19,21,25,30,38-41,44,49,51,52])$.

From the pure mathematical point of view, being able to understand if a tensor is identifiable is a very elegant problem that goes back to Kruskal [45] and finds more modern contributions with the language of Algebraic Geometry and Multilinear Algebra in, e.g. [4,5,11,12,18,22, 23, 26,31,32,34$36,42,50]$. Except for very few contributions $[38,45,46]$ which work for certain specific classes of given tensors, all the others regards the identifiability of generic tensors of certain rank. From the computational point of view, as far as we know, the unique algorithm dealing with the identifiability of any given tensor is a numerical one developed in Bertini [14] in [20].

Dealing with tensors of given rank $r$ brings the problem into the setting of $r$ th secant varieties of Segre varieties (cf. Definition 1.6) namely the closure (either Zariski or Euclidean closures can be used for this definition if working 
over $\mathbb{C}$ ) of the set of tensors of rank smaller or equal than $r$. Knowing if a generic tensor of certain rank is identifiable gives an indication regarding the behaviour of specific tensors of the same rank. Namely, the dimension of the set $\mathcal{S}(Y, T)$ of rank-1 tensors computing the rank of a specific tensor $T$ (cf. Definition 1.4) can only be bigger or equal than the dimension of $\mathcal{S}(Y, q)$ where $q$ is a generic tensor of rank equal to the rank of $T$ (this will be explained in Remark 3.2 for the specific case of rank-3 tensors $T \in\left(\mathbb{C}^{2}\right)^{\otimes 4}$, but it is a well-known general fact for which we refer [43, Cap II, Ex 3.22, part (b)]). Since the cases in which generic tensors of fixed rank are not-identifiable are rare (cf. e.g. [18,22,26-29,34,42,47]), the knowledge of generic tensors' behaviour does not help in all the applied problems where the ken of a specific tensor modeling certain precise samples is required.

In the present manuscript, we present a systematic study of the identifiability of a given tensor starting with those of ranks 2 and 3. We give a complete classification of these first cases: we describe the structures and the dimensions of all the sets evincing the rank. In terms of generic tensors of rank either 2 or 3 , everything was already well-known form $[1,8,27,29,34,36,37,45]$. What it was missing was the complete classification for all the tensors of those ranks.

In Proposition 2.3, we show that rank-2 tensors $T$ are always identifiable except if $T$ is a $2 \times 2$ matrix. Our main Theorem 7.1 states that a rank-3 tensor $T$ is identifiable except if

1. $T$ is a $3 \times 3$ matrix and $\operatorname{dim}(\mathcal{S}(Y, T))=6$;

2. there exist $v_{1}, v_{2}, v_{3} \in \mathbb{C}^{2}$ s.t. $T \in \mathbb{C}^{2} \otimes v_{2} \otimes v_{3}+v_{1} \otimes \mathbb{C}^{2} \otimes v_{3}+v_{1} \otimes v_{2} \otimes \mathbb{C}^{2}$ and $\operatorname{dim}(\mathcal{S}(Y, T)) \geq 2$

3. $T \in\left(\mathbb{C}^{2}\right)^{\otimes 4}$ and $\operatorname{dim}(\mathcal{S}(Y, T)) \geq 1$;

4. $T \in \mathbb{C}^{3} \otimes \mathbb{C}^{2} \otimes \mathbb{C}^{2}$ and it is constructed as in Example 3.6. In this case, $\operatorname{dim}(\mathcal{S}(Y, T))=3$

5. $T \in \mathbb{C}^{3} \otimes \mathbb{C}^{2} \otimes \mathbb{C}^{2}$ and it is constructed as in Example 3.7. In this case, $\mathcal{S}(Y, T)$ contains two different four-dimensional families;

6. $T \in \mathbb{C}^{m_{1}} \otimes \mathbb{C}^{m_{2}} \otimes\left(\mathbb{C}^{2}\right)^{k-2}$, where $k \geq 3$ and $m_{1}, m_{2} \in\{2,3\}$. In this case $\operatorname{dim}(\mathcal{S}(Y, T)) \geq 2$ and $T$ is constructed as in Proposition 3.10. If $m_{1}+m_{2}+k \geq 6$ then $\operatorname{dim}(\mathcal{S}(Y, T))=2$.

The paper is organized as follows. After the preliminary Sect. 1, where we introduce the notation and the main ingredients needed for the set up, we can immediately show the identifiability of rank-2 tensors in Sect. 2. In Sect. 3, we explain in details the examples where the non-identifiability of a rank-3 tensor arises. In Sects. 5 and 6, we show that the examples of the previous section are the only possible exceptions to non-identifiability of a rank-3 tensor. Section 7 is actually devoted to collect all the information needed (but actually already proved at that stage) to conclude the proof of our main Theorem 7.1.

\section{Preliminaries and Notation}

We will always work over an algebraically closed field $\mathbb{K}$ of characteristic 0 . 
Definition 1.1. Let $X \subset \mathbb{P}^{N}$ be a non-degenerate projective variety, the $X$ rank $r_{X}(q)$ of a point $q \in\langle X\rangle$ is the minimal cardinality of a finite set $S \subset X$ such that $q \in\langle S\rangle$.

Notation 1.2. Let $A \subset \mathbb{P}^{N}$ be any subset. With an abuse of notation we denote by $\langle A\rangle$ the projective space spanned by $A$.

Let $V_{1}, \ldots, V_{k}$ be vectors spaces of dimension $n_{1}+1, \ldots, n_{k}+1$, respectively, the Segre variety is the image of the following embedding:

$$
\begin{aligned}
& \nu: \mathbb{P}\left(V_{1}\right) \times \cdots \times \mathbb{P}\left(V_{k}\right) \rightarrow \mathbb{P}\left(V_{1} \otimes \cdots \otimes V_{k}\right) \\
& \quad\left(\left[v_{1}\right], \ldots,\left[v_{k}\right]\right) \mapsto\left[v_{1} \otimes \cdots \otimes v_{k}\right] .
\end{aligned}
$$

Notation 1.3. We denote by $Y$ the multiprojective space

$$
Y:=\mathbb{P}^{n_{1}} \times \cdots \times \mathbb{P}^{n_{k}}
$$

and by $X$ the image of $Y$ via Segre embedding, i.e. $X=\nu(Y)$.

We denote the projection on the $i$ th factor as

$$
\pi_{i}: Y \longrightarrow \mathbb{P}^{n_{i}} \text {. }
$$

The space corresponding to forget the $i$ th factor in the multiprojective space $Y$ is denoted by $Y_{i}$ :

$$
Y_{i}:=\mathbb{P}^{n_{1}} \times \cdots \times \widehat{\mathbb{P}^{n_{i}}} \times \cdots \times \mathbb{P}^{n_{k}} .
$$

With $\nu_{i}: Y_{i} \longrightarrow \mathbb{P}^{N^{\prime}}$ we denote the corresponding Segre embedding, in particular $X_{i}:=\nu\left(Y_{i}\right)$.

The projection on all the factors of $Y$ but the $i$ th one is denoted with $\eta_{i}:$

$$
\eta_{i}: Y \longrightarrow Y_{i}
$$

Obviously all fibers of $\eta_{i}$ are isomorphic to $\mathbb{P}^{n_{i}}$.

Definition 1.4. For any $q \in \mathbb{P}^{N}, \mathcal{S}(Y, q)$ denotes the set of all subsets $A \subset Y$ such that $\sharp(A)=r_{X}(q)$ and $q \in\langle\nu(A)\rangle$ and we will say that if $A \in \mathcal{S}(Y, q)$, then $A$ evinces the rank of $q$. Moreover, we say that $q \in\langle X\rangle$ is identifiable if $\sharp \mathcal{S}(Y, q)=1$.

Notation 1.5. Sometimes we will also use the following multi-index notations: for $1 \leq i \leq k, \varepsilon_{i}=(0, \ldots, 0,1,0, \ldots, 0)$, where the only 1 is in the $i$ th place and $\hat{\varepsilon_{i}}$ which is a $k$-tuple with all one's but the $i$ th place, which is filled by 0 , i.e. $\widehat{\varepsilon_{i}}=(1, \ldots, 1,0,1, \ldots, 1)$.

Definition 1.6. The $r$ th secant variety of $X$ is $\left.\sigma_{r}(X):=\overline{\bigcup_{p, \ldots, p_{r} \in X}\left\langle p_{1}, \ldots, p_{r}\right.}\right\rangle$ where the closure is the the Zariski closure. The set of points of $X$-rank equal to $r$ is sometime denoted as $\sigma_{r}^{0}(X)$. If $\operatorname{dim} \sigma_{r}(X)<\min \{r n+r-1, \operatorname{dim}\langle X\rangle\}$, the variety $X$ is said to be $r$-defective, otherwise $X$ is $r$-regular. If $X$ is $r$ defective, the difference $\delta_{r}=\min \{r n+r-1, \operatorname{dim}\langle X\rangle\}-\operatorname{dim} \sigma_{r}(X)$ is called the $r$ th secant defect of $X$.

We will often use the so called Concision/Autarky property (cf. [48, Prop. 3.1.3.1] [9, Lemma 2.4]) that we recall here. 
Lemma 1.7. (Concision/Autarky) For any $q \in \mathbb{P}\left(V_{1} \otimes \cdots \otimes V_{k}\right)$, there is a unique minimal multiprojective space $Y^{\prime} \simeq \mathbb{P}^{n_{1}^{\prime}} \times \cdots \times \mathbb{P}^{n_{k}^{\prime}} \subseteq Y \simeq \mathbb{P}^{n_{1}} \times \cdots \times$ $\mathbb{P}^{n_{k}}$ with $n_{i}^{\prime} \leq n_{i}, i=1, \ldots, k$ such that $\mathcal{S}(Y, q)=\mathcal{S}\left(Y^{\prime}, q\right)$.

Definition 1.8. (concise Segre) Given a point $q \in \mathbb{P}^{N}$, we will call concise Segre the variety $X_{q}:=\nu\left(Y^{\prime}\right)$ where $Y^{\prime} \subseteq Y$ is the minimal multiprojective space $Y^{\prime} \subseteq Y$ such that $q \in\left\langle\nu\left(Y^{\prime}\right)\right\rangle$ as in Concision/Autarky Lemma 1.7.

Remark 1.9. The minimal $Y^{\prime}$ defining the concise Segre of a point $q$ can be obtained as follows. Fix any $A \in \mathcal{S}(Y, q)$, set $A_{i}:=\pi_{i}(A) \subset \mathbb{P}^{n_{i}}, i=$ $1, \ldots, k$, where the $\pi_{i}$ s are the projections on the $i$ th factor of Notation 1.3. Each $\left\langle A_{i}\right\rangle \subseteq \mathbb{P}^{n_{i}}$ is a well-defined projective subspace of dimension at most $\min \left\{n_{i}, r_{X}(q)-1\right\}$. By Concision/Autarky we have $Y^{\prime}=\prod_{i=1}^{k}\left\langle A_{i}\right\rangle$. In particular $q$ does not depend on the $i$ th factor of $Y$ if and only if for one $A \in \mathcal{S}(Y, q)$ the set $\pi_{i}(A)$ is a single point.

Remark 1.10. Let $q \in \mathbb{P}^{N}$ and consider $A \in \mathcal{S}(Y, q)$. We claim that there is no line $L \subset X$ such that $\sharp(L \cap \nu(A)) \geq 2$. Obviously if $\sharp(L \cap \nu(A))>2$ we would have at least 3 points that evince the rank of $q$ on a line, which is a contradiction with the linearly independence property that sets in $\mathcal{S}(Y, q)$ have. So assume that there exists a line $L \subset X$ such that $\sharp(L \cap \nu(A))=2$; let $u, v \in A$ be the preimages of those points, i.e. $u \neq v$ and $\langle\nu(u), \nu(v)\rangle=L$. Then $r_{X}(q)>2$ because if $r_{X}(q)=2$ then we would have $q \in L \subset X$, so the rank of $q$ will be 1 . Let $E=A \backslash\{u, v\}$. Then we will have that $q \in\langle\nu(E) \cup L\rangle$, so we can find a point $o \in L$ such that $q \in\langle\nu(E) \cup\{o\}\rangle$, which would imply $r_{X}(q)<\sharp A$.

\subsection{A Very Useful Lemma}

Let $X$ be a non degenerate irreducible projective variety embedded in $\mathbb{P}^{N}$ via an ample line bundle $\mathcal{L}$. Let $Z \subset X$ be a zero-dimensional scheme and let $D \subset \mathbb{P}^{N}$ be a fixed hyperplane, i.e. $D \in|\mathcal{L}|$. Denote with $\operatorname{Res}_{D}(Z)$ the residual scheme of $Z$ with respect to $D$, i.e. the zero-dimensional scheme whose defining ideal sheaf is $\mathcal{I}_{Z}: \mathcal{I}_{D}$. The ideal sheaf $\mathcal{I}_{D \cap Z, D} \otimes \mathcal{L}$ represents the scheme theoretic intersection of $D$ and $Z$, also called the trace of $Z$ with respect to $D$. The residual exact sequence of $Z$ with respect to $D$ in $X$ is the following:

$$
0 \rightarrow \mathcal{I}_{\operatorname{Res}_{D}(Z)} \otimes \mathcal{L}(-D) \rightarrow \mathcal{I}_{Z} \otimes \mathcal{L} \rightarrow \mathcal{I}_{D \cap Z, D} \otimes \mathcal{L} \rightarrow 0
$$

An extremely useful tool that will turn out to be crucial in many proofs of this paper is [7, Lemma 5.1]. We recall here the analogous statement given in $[13$, Lemma 2.4] in the setting of zero-dimensional schemes.

Lemma 1.11. (Ballico-Bernardi-Christandl-Gesmundo) Let $X \subseteq \mathbb{P}^{n}$ be an irreducible variety embedded by the complete linear system associated with $\mathcal{L}=\mathcal{O}_{X}(1)$. Let $p \in \mathbb{P}^{n}$ and let $A, B$ be zero-dimensional schemes in $X$ such that $p \in\langle A\rangle, p \in\langle B\rangle$ and there are no $A^{\prime} \subsetneq A$ and $B^{\prime} \subsetneq B$ with $p \in\left\langle A^{\prime}\right\rangle$ or $p \in\left\langle B^{\prime}\right\rangle$. Suppose $h^{1}\left(\mathcal{I}_{B}(1)\right)=0$. Let $C \subseteq \mathbb{P}^{n}$ be an effective Cartier divisor such that $\operatorname{Res}_{C}(A) \cap \operatorname{Res}_{C}(B)=\emptyset$. If $h^{1}\left(X, \mathcal{I}_{\operatorname{Res}_{\mathrm{C}}(\mathrm{A} \cup \mathrm{B})}(1)(-C)\right)=0$ then $A \cup B \subseteq C$. 
We rephrase it in terms of sets of points of multiprojective spaces embedded via $|\mathcal{O}(1, \ldots, 1)|$.

Let $k \geq 2$, let $Y=\mathbb{P}^{n_{1}} \times \cdots \times \mathbb{P}^{n_{k}}$ such that $X:=\nu(Y) \subset \mathbb{P}^{N}$, where $N=\prod\left(n_{i}+1\right)-1$. Let $q \in \mathbb{P}^{N}$ be a point of $X$-rank $r$ and let $A, B \in \mathcal{S}(Y, q)$ be sets of points evincing the rank of $q$ and write $S:=A \cup B$. In this setting, the irreducible variety $X$ considered in Lemma 1.11 is the Segre variety. The residual scheme $\operatorname{Res}_{C}(S)$ is therefore $S \backslash(S \cap C)$. The assumption $h^{1}\left(\mathcal{I}_{B}(1)\right)=0$ of $[13$, Lemma 2.4], in the setting of Segre varieties becomes $h^{1}\left(\mathcal{I}_{B}(1, \ldots, 1)\right)=0$, which means that the points of $\nu(B)$ are linearly independent and this assumption is satisfied since both $A$ and $B$ are sets evincing the rank of $q$.

With all this said we can state the specific version of [13, Lemma 2.4] and [7, Lemma 5.1] which is needed in the present paper.

Notation 1.12. With an abuse of notation, when we will make cohomology computation, if the variety for which we compute the cohomology of the ideal sheaf is $Y$ we will omit it. We will specify the variety only when it is not $Y$.

Lemma 1.13. Let $k \geq 2$ and consider $Y=\mathbb{P}^{n_{1}} \times \cdots \times \mathbb{P}^{n_{k}}$, where all $n_{i} \geq 1$. Let $q \in \mathbb{P}^{N}, A, B \in \mathcal{S}(Y, q)$ be two different subsets evincing the rank of $q$ and write $S=A \cup B$. Let $D \in\left|\mathcal{O}_{Y}(\varepsilon)\right|$ be an effective Cartier divisor such that $A \cap B \subset D$, where $\varepsilon=\sum_{i \in I} \varepsilon_{i}$ for some $I \subset\{1, \ldots, k\}$ as introduced in Notation 1.5. If $h^{1}\left(\mathcal{I}_{S \backslash S \cap D}(\hat{\varepsilon})\right)=0$ then $S \subset D$.

The above lemma gives a sufficient condition so that the whole $S=A \cup B$ is contained in a given divisor $D$ of the variety $X$. If $A, B$ are two disjoint distinct sets evincing the rank of a tensor $q$ of $X$-rank 3 the assumption that $A \cap B \subset D$ is always satisfied.

\section{Identifiability on the 2nd Secant Variety}

In this section we study and completely determine the identifiability of points on the second secant variety of a Segre variety.

By Remark 1.9, the concise Segre of a border rank-2 tensor $q$ is $X_{q}=$ $\nu\left(\mathbb{P}_{i}^{1}\right)^{\times k}$. Therefore, for the rest of this section, we will focus our attention to Segre varieties of products of $\mathbb{P}^{1} \mathrm{~s}$.

Remark 2.1. If the concise Segre $X_{q}$ of a tensor $q \in \sigma_{2}(X)$ is a $\nu\left(\mathbb{P}^{1} \times \mathbb{P}^{1}\right)$, then $\sigma_{2}\left(X_{q}\right)$ parameterizes the $2 \times 2$ matrices for which it is trivial to see that they can be written as sum of two rank-1 matrices in an infinite number of ways.

For the rest of this section, we will, therefore, focus on Segre varieties of $\left(\mathbb{P}^{1}\right)^{\times k}$ with $k \geq 3$.

Definition 2.2. The variety $\tau(X)$ is the tangent developable of a projective variety $X$, i.e. $\tau(X)$ is defined by the union of all tangent spaces to $X$.

Recall that a tensor $q \in \tau(X) \backslash X$ has rank equal to 2 if and only if the concise Segre $X_{q}$ of $q$ is a two-factor Segre; moreover, it is not-identifiable for any number of factors (cf. e.g. [8, Remark 3]). 
Proposition 2.3. Let $q \in \sigma_{2}^{0}(X)$. Then $|\mathcal{S}(Y, q)|>1$ if and only if the concise Segre $X_{q}$ of $q$ is $X_{q}=\nu\left(\mathbb{P}^{1} \times \mathbb{P}^{1}\right)$.

Proof. We only need to check the case of $k \geq 4$ since $k=2,3$ are classically known. The case of matrix is obviously not-identifiable (cf. Remark 2.1), while the identifiability in the case $k=3$ is classically attributed to Segre and it is also among the so called Kruskal range (cf. [45], [36, Thm. 4.6], [34, Thm. 1.2]), see also [37, line 7 of page 484]. We assume, therefore, that $k \geq 4$.

Since $X$ is cut out by quadrics, then if a line $L \subset \mathbb{P}^{N}$ is such that $\operatorname{deg}(L \cap X)>2$ then $L \subset X$ and the points of $L$ have $X$-rank 1. Let $A, B \in$ $\mathcal{S}(Y, q)$, either $\langle A\rangle=\langle B\rangle$ or $\langle A\rangle \cap\langle B\rangle=\{q\}$. In fact, in the first case $A=B$ since $r_{X}(q)=2$ and therefore $\langle A\rangle$ is not contained in $X$; moreover, $X$ is cut out by quadrics. In the second case $A \neq B$. We can, therefore, assume that $A, B \in \mathcal{S}(Y, q)$ are two disjoint sets: $A=\left\{a, a^{\prime}\right\}, B=\left\{b, b^{\prime}\right\}$, where $a=\left(a_{1}, \ldots, a_{k}\right), a^{\prime}=\left(a_{1}^{\prime}, \ldots, a_{k}^{\prime}\right)$ and $b=\left(b_{1}, \ldots, b_{k}\right), b^{\prime}=\left(b_{1}^{\prime}, \ldots, b_{k}^{\prime}\right)$. Since $a \neq a^{\prime}$, we may assume that at least one of their coordinates is different. Actually we can assume that all the $a_{i} \neq a_{i}^{\prime}$, otherwise, by the concision property, one could consider one factor less. The same considerations hold for $B$.

Now suppose that there exists an index $i \in\{1, \ldots, k\}$ such that $\left\{a_{i}, a_{i}^{\prime}\right\} \neq$ $\left\{b_{i}, b_{i}^{\prime}\right\}$ and let such an index be $i=1:\left\{a_{1}, a_{1}^{\prime}\right\} \neq\left\{b_{1}, b_{1}^{\prime}\right\}$.

Now we proceed by induction on $k$. Let $\eta_{k}, \nu_{k}$, and $X_{k}$ be as in Notation 1.3. Let $\tilde{q}=\left(q_{1}, \ldots, q_{k-1}\right)$ be the projection $\eta_{k}(q)$, then $\eta_{k}(A) \neq \eta_{k}(B)$ and $\emptyset \neq\left\langle\nu_{k}\left(\eta_{k}(A)\right)\right\rangle \cap\left\langle\nu_{k}\left(\eta_{k}(B)\right)\right\rangle \supset\{\tilde{q}\}$ because $\{q\} \subset\langle\nu(A)\rangle \cap\langle\nu(B)\rangle$. So $r_{X_{k}}(\tilde{q})=2$ and $\left|\mathcal{S}\left(Y_{k}, \tilde{q}\right)\right| \geq 2$, which is a contradiction because $X_{k}$ is a concise Segre of $k-1$ factor (where $k>3$ ) and a point of it cannot have more than a decomposition. Thus, for all $i=, 1 \ldots, k$ we have that $\left\{a_{i}, a_{i}^{\prime}\right\}=\left\{b_{i}, b_{i}^{\prime}\right\}$.

Without loss of generality assume that $a_{1}=b_{1}$ and $a_{1}^{\prime}=b_{1}^{\prime}$, moreover up to permutation there exists an index $e \in\{1, \ldots, k-1\}$ such that $b_{i}=a_{i}$ and consequently $b_{i}^{\prime}=a_{i}^{\prime}$ for $1 \leq i \leq e$ and $b_{i}=a_{i}^{\prime}$ and $b_{i}^{\prime}=a_{i}$ for $e+1 \leq i \leq k$. Eventually by exchanging the role of the first $e$ elements with the others, we have that $k-e \geq 2$ because by assumption $k \geq 4$. Let $H \in\left|\mathcal{O}_{Y}(0, \ldots, 0,1)\right|$ be the only element containing $a^{\prime}, H=\mathbb{P}^{1} \times \cdots \times \mathbb{P}^{1} \times\left\{a_{k}^{\prime}\right\} \cong\left(\mathbb{P}^{1}\right)^{\times k-1}$; then $\operatorname{Res}_{H}(A \cup B)=\left\{a^{\prime}, b^{\prime}\right\}$ and since $k-e \geq 2$ we have that $\eta_{k}\left(a^{\prime}\right) \neq \eta_{k}\left(b^{\prime}\right)$, i.e. $h^{1}\left(\mathcal{I}_{\operatorname{Res}_{H}(A \cup B)}(1, \ldots, 1,0)\right)=0$. By Lemma 1.13 , we get $a^{\prime}=b^{\prime}$ which contradicts the fact that $A \cap B=\emptyset$.

Corollary 2.4. Let $q$ be any rank-2 tensor. If $q$ is not-identifiable, then there is a bijection between $\mathcal{S}(Y, q)$ and $\mathbb{P}^{2} \backslash L$, where $L \subset \mathbb{P}^{2}$ is a projective line, $q \in \tau(X)$ and $L$ parametrizes the set of all degree 2 connected subschemes $V$ of $Y$ such that $q \in\langle\nu(V)\rangle$.

Proof. It suffices to work with a Segre variety of 2 factors only because by Proposition 2.3 it is the only not-identifiable case in rank-2. Thus, $X \subset \mathbb{P}^{3}$ is a quadric surface. Denote by $H_{q} \subset \mathbb{P}^{3}$ the polar plane of $X$ with respect to $q$. Since $q \notin X$, we have that $q \notin H_{q}$ and the intersection $X \cap H_{q}=\{p \in$ $\left.X \mid T_{p} X \ni q\right\}$ is a smooth conic. Remark also that by definition a point $o \in X$ is such that $q \in T_{o} X$ if and only if $o \in X \cap H_{q} \subset \tau(X)$.

Fix $o \in H_{q}$, then 
- if $o \notin X$ the line given by $\langle o, q\rangle$ is not tangent to $X$ and when considering the intersection $\langle o, q\rangle \cap X$, it is given by two points $p_{1}, p_{2} \notin\{o, q\}$ such that $\left\{p_{1}, p_{2}\right\} \in \mathcal{S}(Y, q)$;

- if $o \in X$, i.e. $o \in X \cap H_{q}$, then the line $\langle o, q\rangle$ is tangent to $X$.

Consider $\Pi_{q}=\left\{\right.$ lines $L \subset \mathbb{P}^{3}$ passing through $\left.q\right\} \cong \mathbb{P}^{2}$ and consider the following isomorphism $\varphi: H_{q} \longrightarrow \Pi_{q}$ defined by $p \mapsto\langle p, q\rangle$. Clearly, $\varphi(X \cap$ $\left.H_{q}\right)$ is a smooth conic $\mathcal{C}$ of $\Pi_{q}$. Moreover, one can notice that $\Pi_{q} \backslash \varphi\left(X \cap H_{q}\right) \cong$ $\mathbb{P}^{2} \backslash \mathcal{C}$ are just the points of the first case.

\section{Examples of Not-Identifiable Rank-3 Tensors}

The purpose of this section is to explain in detail the phenomena behind the not-identifiable rank-3 tensors. In the main Theorem 7.1 , they will turn out to be the unique cases of not-identifiability for a rank-3 tensor.

From now on, we always consider $q \in \mathbb{P}^{N}$ such that $r_{X}(q)=3$; therefore, by Remark 1.9, we may assume that $q$ is an order- $k$ tensor with at most 3 entries in each mode, i.e. the concise Segre of $q$ is $X_{q}=\nu\left(\mathbb{P}^{n_{1}} \times \cdots \times \mathbb{P}^{n_{k}}\right)$, with $n_{1}, \ldots, n_{k} \in\{1,2\}$.

First of all let us remark that the matrix case is highly not-identifiable even for the rank-3 case.

Remark 3.1. In the case of two factors (i.e. $k=2$ ), a rank-3 tensor $q$ is a $3 \times 3$ matrix of full rank. The dimension of the concise Segre $X$ of $3 \times 3$ matrices is 4 and $\operatorname{dim}\left(\sigma_{3}(X)\right)=\min \left\{\operatorname{dim}\left(\mathbb{P}^{8}\right), 3 \operatorname{dim}(X)+2\right\}=\min \{8,14\}=8$. Thus, $\operatorname{dim} \mathcal{S}(Y, q)=14-8=6$ for all $q \in \mathbb{P}^{8}$ of rank 3 .

Consider now the third secant variety of the Segre embedding of $Y=$ $\mathbb{P}^{n_{1}} \times \cdots \times \mathbb{P}^{n_{k}}$, where $n_{i} \in\{1,2\}$, the following Examples 3.6 and 3.7 and Proposition 3.10 provides instances of not-identifiability that we will show to be essentially the only classes of not-identifiable rank-3 tensors in $\mathbb{C}^{n_{1}+1} \otimes$ $\cdots \otimes \mathbb{C}^{n_{k}+1}$ (cases (4), (5) and (6), respectively, of our main Theorem 7.1 ) more than the well-known ones (matrix case, points on tangential variety of $\nu\left(\left(\mathbb{P}^{1}\right)^{\times 3}\right)$, and elements of the defective $\sigma_{3}\left(\nu\left(\left(\mathbb{P}^{1}\right)^{\times 4}\right)\right)$-items $(1),(2)$ and (3) respectively of Theorem 7.1 ).

In the following remark we explain the behaviour on $\sigma_{3}\left(\left(\mathbb{P}^{1}\right)^{\times 4}\right)$.

Remark 3.2. It has been shown in [1] (cf. also [27,29]) that the third secant variety of a Segre variety $X$ is never defective unless either $X=\nu\left(\mathbb{P}^{1} \times \mathbb{P}^{1} \times\right.$ $\left.\mathbb{P}^{1} \times \mathbb{P}^{1}\right)$ or $X=\nu\left(\mathbb{P}^{1} \times \mathbb{P}^{1} \times \mathbb{P}^{a}\right)$, with $a \geq 3$.

The case in which $q$ is a rank-3 tensor in $\left\langle\nu\left(\mathbb{P}^{1} \times \mathbb{P}^{1} \times \mathbb{P}^{a}\right)\right\rangle$ with $a \geq 3$ corresponds to a not-concise tensor (cf. Remark 1.9); therefore, it will not play a role in our further discussion.

The case in which $X=\nu\left(\mathbb{P}^{1} \times \mathbb{P}^{1} \times \mathbb{P}^{1} \times \mathbb{P}^{1}\right)$ and $q \in\langle X\rangle$ can also be easily handled. The fact that $\operatorname{dim}\left(\sigma_{3}(X)\right)$ is strictly smaller than the expected dimension proves that the generic element of $\sigma_{3}(X)$ has an infinite number of rank-3 decompositions. By definition of dimension there is no element of $\sigma_{3}(X)$ s.t. its tangent space has dimension equal to the expected one: $\operatorname{dim}\left(T_{q}\left(\sigma_{3}(X)\right)\right) \leq \operatorname{dim} \sigma_{3}(X)$ for all $q \in \sigma_{3}(X)$. This does not exclude the 
existence of certain special rank-3 tensors $q$ such that $\operatorname{dim}\left(T_{q}\left(\sigma_{3}(X)\right)\right)=$ $\operatorname{dim}\left(T_{q^{\prime}}\left(\operatorname{AbSec}_{3}(X)\right)\right)<14$ where $\operatorname{AbSec}_{3}(X):=\left\{\left(p ; p_{1}, p_{2}, p_{3}\right) \in \mathbb{P}^{15} \times\right.$ $\left.X^{\times 3} \mid p \in\left\langle p_{1}, p_{2}, p_{3}\right\rangle\right\}$ is the 3rd abstract secant of $X$ and $q^{\prime}$ is the preimage of $q$ via the projection on the first factor. The impossibility of the existence of such a point is guaranteed by [43, Cap II, Ex 3.22, part (b)]. This proves that all the tensors of $\sigma_{3}^{0}(X)$ have an infinite number of rank-3 decompositions.

Before explaining the other not-identifiable examples, we need some preliminary results.

Remark 3.3. Let $Y$ be a multiprojective space with at least two factors where at least one of them is of projective dimension 2. By relabeling, if necessary, we can assume that the first factor is a $\mathbb{P}^{2}$. Let $q \in \sigma_{3}^{0}(\nu(Y))$, with $\nu(Y)$ the concise Segre of $q$ and let $A, B \in \mathcal{S}(Y, q)$ be two disjoint subsets evincing the rank of $q$. By Autarky $\left\langle\pi_{1}(A)\right\rangle=\left\langle\pi_{1}(B)\right\rangle=\mathbb{P}^{2}$; moreover when considering the restrictions of the projections $\pi_{1 \mid A}$ and $\pi_{1 \mid B}$ to the subsets $A$ and $B$, respectively; they are both injective and both $\pi_{1}(A)$ and $\pi_{1}(B)$ contain linearly independent points.

Remark 3.4. Consider $Y=\mathbb{P}^{2} \times \mathbb{P}^{1} \times \mathbb{P}^{1}$ and an irreducible divisor $G \in$ $\left|\mathcal{O}_{Y}(0,1,1)\right|$. Then $\sigma_{2}(\nu(G)) \subsetneq \sigma_{3}(\nu(G))=\langle\nu(G)\rangle=\mathbb{P}^{8}$. Indeed $G$ is nothing else than the Segre-Veronese variety $([16])$ of $\mathbb{P}^{2} \times \mathbb{P}^{1}$ embedded in bidegree $(1,2)$, i.e. $G \cong \mathbb{P}^{2} \times \mathbb{P}^{1}, \mathcal{O}_{Y}(1,1,1)_{\left.\right|_{G}} \cong \mathcal{O}_{\mathbb{P}^{2} \times \mathbb{P}^{1}}(1,2)$ and $\mathcal{O}_{Y}(1,0,0) \cong$ $\mathcal{O}_{Y}(1,1,1)(-G)$. The classification of the dimensions of secant varieties of such a Segre-Veronese can be found in $[10,15,17,33]$.

Proposition 3.5. For the Segre embedding of $Y=\mathbb{P}^{2} \times \mathbb{P}^{1} \times \mathbb{P}^{1}$ fix $G_{1} \in$ $\left|\mathcal{O}_{Y}(0,1,0)\right|$ and $G_{2} \in\left|\mathcal{O}_{Y}(0,0,1)\right|$ and define $G:=G_{1} \cup G_{2}$ to be their union. We have that for $\{i, j\}=\{1,2\}, \operatorname{dim}\left\langle\nu\left(G_{i}\right)\right\rangle=5, \operatorname{dim}\langle\nu(G)\rangle=8$, $\sigma_{2}\left(\nu\left(G_{i}\right)\right)=\left\langle\nu\left(G_{i}\right)\right\rangle$ and $\langle\nu(G)\rangle$ is the join of $\sigma_{2}\left(\nu\left(G_{i}\right)\right)$ and $\nu\left(G_{j}\right)$.

Proof. First of all remark that, for $i=1,2, G_{i} \cong \mathbb{P}^{2} \times \mathbb{P}^{1}, \mathcal{O}_{Y}(1,1,1)_{\left.\right|_{i}} \cong$ $\mathcal{O}_{\mathbb{P}^{2} \times \mathbb{P}^{1}}(1,1)$ and $G$ is a reducible element of $\left|\mathcal{O}_{Y}(0,1,1)\right|$. With an analogous computation of the one in Remark 3.4 one sees that $\operatorname{dim}\langle\nu(G)\rangle=8$ and $\sigma_{2}\left(\nu\left(G_{i}\right)\right)=\left\langle\nu\left(G_{i}\right)\right\rangle$. It remains to show that $\langle\nu(G)\rangle=\mathcal{J}$, where $\mathcal{J}$ denotes the join of $\sigma_{2}\left(\nu\left(G_{i}\right)\right)$ and $\nu\left(G_{j}\right)$ with $\{i, j\}=\{1,2\}$. We remark that since $\sigma_{2}(\nu(G))=\mathbb{P}^{5}$, then $\mathcal{J}=\operatorname{Join}\left(\mathbb{P}^{3}, \nu\left(G_{1}\right), \nu\left(G_{2}\right)\right)$. To show that $\mathcal{J}=\mathbb{P}^{8}$ it is sufficient to see that $\operatorname{dim}\left(\sigma_{2}\left(\nu\left(G_{i}\right) \cap \nu\left(G_{j}\right)\right)\right)=1$ and this is a straightforward computation since the elements of $\nu\left(G_{1}\right)$ are tensors with a second factor fixed, while the elements of $\nu\left(G_{2}\right)$ have the third factor fixed, and to have the equality between an element of $\sigma_{2}\left(\nu\left(G_{1}\right)\right)$ and an element of $\nu\left(G_{2}\right)$ it is sufficient to impose two linear independent conditions and, therefore, since $\operatorname{dim}\left(\nu\left(G_{2}\right)\right)=3$ we have that the intersection has dimension 1 .

Example 3.6. Take $Y=\mathbb{P}^{2} \times \mathbb{P}^{1} \times \mathbb{P}^{1}$, consider the Segre embedding on the last two factors and take a hyperplane section which intersects $\nu\left(\mathbb{P}^{1} \times \mathbb{P}^{1}\right)$ in a conic $\mathcal{C}$, then take a point $q \in\left\langle\nu\left(\mathbb{P}^{2} \times \mathcal{C}\right)\right\rangle$. Such a construction is equivalent to consider an irreducible divisor $G \in\left|\mathcal{O}_{Y}(0,1,1)\right|$, so $G \cong \mathbb{P}^{2} \times \mathbb{P}^{1}$ embedded via $\mathcal{O}(1,2)$, then $\operatorname{dim} \sigma_{2}(\nu(G))=7$, thus $\sigma_{2}(\nu(G)) \subsetneq\langle\nu(G)\rangle \simeq \mathbb{P}^{8}$. As a direct consequence we get that a general point $q \in\langle\nu(G)\rangle$ has $\nu(G)$-rank 3 and it 
is not-identifiable because of the not-identifiability of the points on $\langle\mathcal{C}\rangle$ and by [43, Cap II, Ex 3.22, part (b)]. Thus, $\operatorname{dim}(\mathcal{S}(G, q))=3$.

The following example is in the same setting of the previous one, but in this case we deal with a reducible conic and in such a case we get a 4dimensional family of solutions.

Example 3.7. Fix $Y=\mathbb{P}^{2} \times \mathbb{P}^{1} \times \mathbb{P}^{1}$. Consider $G_{1} \in\left|\mathcal{O}_{Y}(0,0,1)\right|, G_{2} \in$ $\left|\mathcal{O}_{Y}(0,1,0)\right|$ and call $G=G_{1} \cup G_{2}$ which is a reducible element of $\left|\mathcal{O}_{Y}(0,1,1)\right|$. By Proposition 3.5, $\operatorname{dim}\langle\nu(G)\rangle=8$; moreover, by a dimension count, we have $\left\langle\nu\left(G_{i}\right)\right\rangle=\sigma_{2}\left(G_{i}\right)$, for $i=1,2$, both having dimension 5. By Proposition 3.5, we also have that $\langle\nu(G)\rangle=\mathcal{J}_{1}=\mathcal{J}_{2}$, where $\mathcal{J}_{1}=\operatorname{Join}\left(\sigma_{2}\left(\nu\left(G_{1}\right)\right), \nu\left(G_{2}\right)\right)$ and $\mathcal{J}_{2}=\operatorname{Join}\left(\sigma_{2}\left(\nu\left(G_{2}\right)\right), \nu\left(G_{1}\right)\right)$. A general $q \in\langle\nu(G)\rangle$ has rank 3 and for the subsets evincing its rank we have a 4-dimensional family of sets $A$ such that $\sharp(A)=3, \sharp\left(A \cap G_{1}\right)=2, \sharp\left(A \cap G_{2}\right)=1, A \cap G_{1} \cap G_{2}=\emptyset$ and $q \in\langle\nu(A)\rangle$. Such a family has dimension 4 since $G_{1}$ is a non defective threefold in $\mathbb{P}^{5}$; therefore, there exists a 2-dimensional family of sets of cardinality 2 in $G_{1}$ spanning a general point of $\mathbb{P}^{5}$; moreover, $q$ sits in a 2-dimensional family of lines joining points of $G_{1}$ and $G_{2}$. Analogously, by looking at $q$ as an element of $\mathcal{J}_{2}$, we get the existence of a 4 -dimensional family of sets $B$ such that $\sharp(B)=3, \sharp\left(B \cap G_{2}\right)=2, \sharp\left(B \cap G_{1}\right)=1, A \cap G_{1} \cap G_{2}=\emptyset$ and $q \in\langle\nu(B)\rangle$. So we proved that $\mathcal{S}(G, q)$ contains at least two dimensional families of solution. Thus, $\operatorname{dim} \mathcal{S}(G, q) \geq 4$.

Proposition 3.8. Let $q \in \sigma_{3}^{0}\left(\nu\left(\mathbb{P}^{2} \times \mathbb{P}^{1} \times \mathbb{P}^{1}\right)\right)$ and suppose that there exist $A, B \in \mathcal{S}(Y, q)$ s.t. $\sharp(A \cup B)=6$. Then there exists a unique $G \in\left|\mathcal{O}_{Y}(0,1,1)\right|$ containing $S=A \cup B$. For such a $G$ we have that $\mathcal{S}(Y, q)=\mathcal{S}(G, q)$.

Proof. Call $S:=A \cup B$, by Remark 3.3, both $\pi_{1 \mid A}$ and $\pi_{1 \mid B}$ are injective and both $\pi_{1}(A)$ and $\pi_{1}(B)$ are sets containing linearly independent points. So $h^{1}\left(\mathcal{I}_{A}(1,0,0)\right)=h^{1}\left(\mathcal{I}_{B}(1,0,0)\right)=0$. Now $h^{0}\left(\mathcal{O}_{Y}(0,1,1)\right)=4$, so there exists $G \in\left|\mathcal{O}_{Y}(0,1,1)\right|$ containing $B$. Moreover, $S \backslash S \cap G \subseteq A$ but since $h^{1}\left(\mathcal{I}_{A}(1,0,0)\right)=0$ we have that $S \subset G$. This holds for any $G \in\left|\mathcal{I}_{B}(0,1,1)\right|$, so $\left\langle\nu_{1}\left(\eta_{1}(A)\right)\right\rangle \subset\left\langle\nu_{1}\left(\eta_{1}(B)\right)\right\rangle$. The same holds exchanging the roles of $A$ and $B$; thus, $\left\langle\nu_{1}\left(\eta_{1}(A)\right)\right\rangle=\left\langle\nu_{1}\left(\eta_{1}(B)\right)\right\rangle$.

Assume $G$ is irreducible, then $B$ contains three linearly independent points on $G$, so the points of $B$ are uniquely determined by $G$.

Assume $G$ is reducible, i.e. $G=G_{1} \cup G_{2}$, with $G_{1} \in\left|\mathcal{O}_{Y}(0,1,0)\right|$ and $G_{2} \in\left|\mathcal{O}_{Y}(0,0,1)\right|$. Remark that, by Autarky, it does not exist any $E \in \mathcal{S}(Y, q)$ which is all contained in $G_{i}$, for $i=1,2$, because $G$ is a multiprojective subspace of $Y$.

Without loss of generality, we may assume that two points of $E$ lies in $G_{1}$; then the three points of $E$ are uniquely determined by a reducible conic, i.e. by the reducible element $G=G_{1} \cup G_{2}$ that contains them.

Corollary 3.9. If $q \in \sigma_{3}^{0}\left(\nu\left(\mathbb{P}^{2} \times \mathbb{P}^{1} \times \mathbb{P}^{1}\right)\right)$ is such that there exist two disjoint sets $A, B \in \mathcal{S}(Y, q)$, then $q$ can be either as in Example 3.6 and $\operatorname{dim}(\mathcal{S}(Y, q))=$ 3 or as in Example 3.7 and $\operatorname{dim}(\mathcal{S}(Y, q))=4$. 
Proof. This is a direct consequence of the uniqueness of the $G \in\left|\mathcal{O}_{Y}(0,1,1)\right|$ s.t. $\mathcal{S}(Y, q)=\mathcal{S}(G, q)$ in Proposition 3.8.

Proposition 3.10. Let $Y^{\prime}:=\mathbb{P}^{1} \times \mathbb{P}^{1} \times\left\{u_{3}\right\} \times \cdots \times\left\{u_{k}\right\}$ be a proper subset of $Y=\mathbb{P}^{n_{1}} \times \cdots \times \mathbb{P}^{n_{k}}, k \geq 2$. Take $q^{\prime} \in\left\langle\nu\left(Y^{\prime}\right)\right\rangle \backslash \nu\left(Y^{\prime}\right), A \in \mathcal{S}\left(Y^{\prime}, q^{\prime}\right)$ and $p \in Y \backslash Y^{\prime}$. Assume that $Y$ is the minimal multiprojective space containing $A \cup\{p\}$ and take $q \in\left\langle\left\{q^{\prime}, \nu(p)\right\}\right\rangle \backslash\left\{q^{\prime}, \nu(p)\right\}$.

1. $\sum_{i=1}^{k} n_{i} \geq 3, n_{1}, n_{2} \leq 2, n_{3}, \ldots, n_{k} \leq 1$ and if $k \geq 3$ then $r_{\nu(Y)}(q)>1$;

2. If $k \geq 3$ and $\sum_{i=1}^{k} n_{i} \geq 4$ then $r_{\nu(Y)}(q)=3$ and $\mathcal{S}(Y, q)=\{\{p\} \cup$ $A\}_{A \in \mathcal{S}\left(Y^{\prime}, q^{\prime}\right)}$.

3. $\nu(Y)$ is the concise Segre of $q$.

Proof. First of all, remark that $r_{\nu(Y)}(q)>1$, otherwise there exists $o \in Y$ s.t. $q=\nu(o)$ and $q^{\prime} \in\langle\nu(\{o, p\})\rangle$. Since $r_{\nu(Y)}\left(q^{\prime}\right)=2$, we would have $\{o, p\} \in$ $\mathcal{S}\left(Y, q^{\prime}\right)$ and by Autarky we get $\{o, p\} \subset Y^{\prime}$, contradicting the assumption $p \notin Y^{\prime}$. $Y \neq Y^{\prime}$.

The fact that $n_{1}+\cdots+n_{k} \geq 3$ is obvious from the fact that $p \notin Y^{\prime}$ so

Since $q^{\prime}$ is a $2 \times 2$ matrix of rank $2, \operatorname{dim} \mathcal{S}\left(Y^{\prime}, q^{\prime}\right)=2$ and $Y^{\prime}$ is the minimal multiprojective subspace of $Y$ containing $A$, the minimal multiprojective subspace containing $Y^{\prime} \cup\{p\}$ is $Y$. So since $\mathbb{P}^{n_{i}}=\left\langle\pi_{i}\left(Y^{\prime} \cup\{p\}\right)\right\rangle$, we get $1 \leq n_{i} \leq 2$ for $i=1,2$ and $n_{i}=1$ for all $i>2$. This ends item 1 .

Item 3 will be a consequence of item 2 , in fact if the structure of the elements on $\mathcal{S}(Y, q)$ is of type $A \cup\{p\}$ with $A \in \mathcal{S}\left(Y^{\prime}, q^{\prime}\right)$, then Autarky and the fact that $Y$ is the minimal multiprojective space containing $A \cup\{p\}$ will imply that $\nu(Y)$ is the concise Segre of $q$. So let us prove item 2.

The proof is by induction on the number of factors. Step (A) is the basis of induction for the case in which $Y$ has at least one factor of projective dimension $2(k=3)$, Step $(\mathrm{B})$ is the basis of induction for the case in which all the factors of $Y$ have projective dimension $1(k=4)$, Steps (C) and (D) are the induction processes of Step (B) and Step (A), respectively.

Let $E \in \mathcal{S}(Y, q)$, if we will show that $E \supset\{p\}$ and that there exists $B \in \mathcal{S}\left(Y^{\prime}, q^{\prime}\right)$, such that $E=B \cup\{p\}$, we will be done. Assume that there is no $B \in \mathcal{S}\left(Y^{\prime}, q^{\prime}\right)$ such that $E=B \cup\{p\}$. Fix any $A \in \mathcal{S}\left(Y^{\prime}, q^{\prime}\right)$ and set $S:=A \cup\{p\} \cup E$.

(A) [Case $k=3, n_{1}=2, n_{2}=n_{3}=1$ ] First assume $p \in E$ and set $E^{\prime}$ : $=E \backslash\{p\}$ and $F=A \cup E^{\prime}$. Since $\cap_{B \in \mathcal{S}\left(Y, q^{\prime}\right)} \eta_{3}(B)=\emptyset$, taking another $A \in \mathcal{S}\left(Y, q^{\prime}\right)$ if necessary we may assume $\eta_{3}(A) \cap \eta_{3}\left(E^{\prime}\right)=\emptyset$. Set $\{D\}:=\left|\mathcal{I}_{p}(0,0,1)\right|$. By Lemma 1.13 , we have $h^{1}\left(\mathcal{I}_{S \backslash S \cap D}(1,1,0)\right)>0$ and hence (since $\sharp F \leq 4) h^{0}\left(\mathcal{I}_{S \backslash S \cap D}(1,1,0)\right) \geq 3$. This must be true for all $A \in \mathcal{S}\left(Y^{\prime}, q^{\prime}\right)$ and hence we have $h^{0}\left(Y_{3}, \mathcal{I}_{\eta_{3}\left(Y^{\prime}\right) \cup \eta_{3}\left(E^{\prime}\right)}(1,1)\right) \geq 3$. Since $\eta_{3}\left(Y^{\prime}\right) \in\left|\mathcal{O}_{Y_{3}}(1,1)\right|$ we have $h^{0}\left(Y_{3}, \mathcal{I}_{\eta_{3}\left(Y^{\prime}\right)}(1,1)\right)=1$, contradicting the previous inequality.

From now on suppose $p \notin E$. As above we may assume $\eta_{3}(A) \cap \eta_{3}(E)=$ $\emptyset$.

Fix $o \in E$. Since $h^{0}\left(\mathcal{O}_{Y}(1,1,0)\right)=6$ and $\sharp A \cup\{p\} \cup\{o\}=4$ there is $G \in\left|\mathcal{O}_{Y}(1,1,0)\right|$ containing $A \cup\{p\} \cup\{o\}$. Assume for the moment 
$S \nsubseteq G$, i.e. $E \nsubseteq G$. We have $h^{1}\left(\mathcal{I}_{S \backslash S \cap G}(0,0,1)\right)>0$; thus, $\sharp E \geq 3$. Since $\sharp E \leq 3$, we get $\sharp E=3$ (and hence $q$ has rank 3 and $\nu(Y)$ is the concise Segre containing $q), S \backslash S \cap G=E \backslash\{o\}$ and $\sharp \pi_{3}(E \backslash\{o\})=1$. Taking a different $o \in E$ we get $\sharp \pi_{3}(E)=1$, i.e. $\nu(Y)$ is not the concise Segre of $q$, a contradiction.

Now assume $S \subset G$. Since this must be true for all $G \in\left|\mathcal{I}_{A \cup\{p, o\}}(1,1,0)\right|$, we get $\left|\mathcal{I}_{A \cup\{p, o\}}(1,1,0)\right| \supseteq\left|\mathcal{I}_{\{p\} \cup E}(1,1,0)\right| \neq \emptyset$. Note that $\eta_{3}\left(Y^{\prime}\right) \in$ $\left|\mathcal{O}_{Y_{3}}(1,1)\right|$ and hence $h^{0}\left(Y_{3}, \mathcal{I}_{\eta_{3}\left(Y^{\prime}\right)}(1,1)\right)=1$. Since $n_{1}=2$ and $Y$ is the minimal multiprojective space containing $q$, we have $\eta_{3}(p) \notin$ $\eta_{3}\left(Y^{\prime}\right)$. Thus, $h^{0}\left(Y_{3}, \mathcal{I}_{\eta_{3}\left(Y^{\prime}\right) \cup\left\{\eta_{3}(p)\right\}}(1,1)\right)=0$, a contradiction since $\left|\mathcal{I}_{A \cup\{p, o\}}(1,1,0)\right| \neq \emptyset$.

(B) $\left[\right.$ Case $\left.k=4, n_{1}=n_{2}=n_{3}=n_{4}=1\right]$ Fix $G \in\left|\mathcal{O}_{Y}(0,0,1,1)\right|$ containing $E$. Assume $S \nsubseteq G$. Since $S \backslash E=A \cup\{p\}$, by Lemma 1.13, we have $h^{1}\left(\mathcal{I}_{A \cup\{p\}}(1,1,0,0)\right)>0$. Call $p^{\prime}$ the projection of $p$ via $Y \rightarrow Y^{\prime}$. Since $\mathcal{O}_{\mathbb{P}^{1} \times \mathbb{P}^{1}}(1,1)$ is very ample we get that either $p^{\prime} \in A$ or that $\sharp\left(\pi_{i}\left(A \cup\left\{p^{\prime}\right\}\right)\right)=1$ for some $i \in\{1,2\}$. The second possibility is excluded, because $\sharp\left(\pi_{1}(A)\right)=\sharp\left(\pi_{2}(A)\right)=2$ for any $A \in \mathcal{S}\left(Y^{\prime}, q^{\prime}\right)$. The first possibility is excluded taking instead of $A$ another general $A_{1} \in \mathcal{S}\left(Y^{\prime}, q^{\prime}\right)$. Now assume $S \subset G$. We get $A \subset G$. This is ruled out taking another $A \in \mathcal{S}\left(Y^{\prime}, q^{\prime}\right)$ since a general $a \in Y^{\prime}$ is contained in some $B \in \mathcal{S}\left(Y^{\prime}, q^{\prime}\right)$. Thus, we would have that $Y^{\prime} \subset G$ which is a contradiction.

(C) [Case $k \geq 5, n_{i}=1$ for all $i$ s] We exclude this case by induction on $k$, the base case $k=4$ being excluded in (B). Fix $o \in \mathbb{P}^{1} \backslash\left\{p_{k}, u_{k}\right\}$, set $M:=\pi_{k}^{-1}(o)$, i.e. $M=\left(\mathbb{P}^{1}\right)^{\times k-1} \times\{o\}$ and call $\Lambda:=\langle\nu(M)\rangle$. Note that $\left(Y^{\prime} \cup\{p\}\right) \cap M=\emptyset$. Denote by $r=2^{k}-1$ and define $r^{\prime}:=\operatorname{dim} \Lambda=$ $2^{k-1}-1$.

Consider the following linear projection form $\Lambda$ :

$$
\ell: \mathbb{P}^{r} \backslash \Lambda \rightarrow \mathbb{P}^{r^{\prime}}
$$

Note that $\nu(Y) \cap \Lambda=\nu\left(Y_{k}\right) \times\{o\}$ and that $\ell_{\mid \nu(Y) \backslash M}=\nu_{k}\left(\eta_{k}(Y \backslash M)\right)$. We identify $\mathbb{P}^{r^{\prime}}$ with the target projective space of $Y_{k}$. Since $\left(Y^{\prime} \cup\{p\}\right) \cap M=$ $\emptyset, \ell$ is well defined on $Y^{\prime} \cup\{p\}$ and it acts as the composition of $\eta_{k}$ and the Segre embedding.

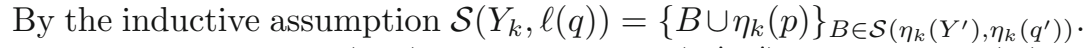
Thus, for any $E \in \mathcal{S}(Y, q)$ there is $B \in \mathcal{S}\left(Y^{\prime}, q^{\prime}\right)$ such that $\eta_{k}(E)=$ $\eta_{k}(B \cup\{p\})$. Since $\eta_{k \mid E}$ is injective by Remark 1.10 and $\mathcal{S}(Y, q) \supseteq\{B \cup$ $\{p\}\}_{B \in \mathcal{S}\left(Y^{\prime}, q^{\prime}\right)}$, we get $\mathcal{S}(Y, q)=\{B \cup\{p\}\}_{B \in \mathcal{S}\left(Y^{\prime}, q^{\prime}\right)}$.

(D) [Case $\left.k \geq 3, n_{1}=2, n_{1}+\cdots+n_{k} \geq 5\right]$ If only one of the factors is a $\mathbb{P}^{2}$ we use Step (A) as base of the induction and then we construct a projection similar to (3.1). Indeed $Y=\mathbb{P}^{2} \times\left(\mathbb{P}^{1}\right)^{k-1}$, where $k \geq 4$. Fix $o \in \mathbb{P}^{1} \backslash\left\{p_{k}, u_{k}\right\}$, set $M:=\pi_{k}^{-1}(o)$ and define $\Lambda:=\langle\nu(M)\rangle$. Denote by $r=3 \cdot 2^{k-1}-1$ and by $r^{\prime}=\operatorname{dim} \Lambda:=3 \cdot 2^{k-2}-1$. We consider the linear projection $\ell: \mathbb{P}^{r} \backslash \Lambda \rightarrow \mathbb{P}^{r^{\prime}}$ which acts as the composition of $\eta_{k}$ and the Segre embedding. By the inductive assumption $\mathcal{S}\left(Y_{k}, \ell(q)\right)=\{B \cup$ $\left.\eta_{k}(p)\right\}_{B \in \mathcal{S}\left(\eta_{k}\left(Y^{\prime}\right), \eta_{k}\left(q^{\prime}\right)\right)}$. Thus, for any $E \in \mathcal{S}(Y, q)$ there is $B \in \mathcal{S}\left(Y^{\prime}, q^{\prime}\right)$ 
such that $\eta_{k}(E)=\eta_{k}(B \cup\{p\})$. Since $\eta_{k \mid E}$ is injective by Remark 1.10 and $\mathcal{S}(Y, q) \supseteq\{B \cup\{p\}\}_{B \in \mathcal{S}\left(Y^{\prime}, q^{\prime}\right)}$, we get $\mathcal{S}(Y, q)=\{B \cup\{p\}\}_{B \in \mathcal{S}\left(Y^{\prime}, q^{\prime}\right)}$. Now assume also $n_{2}=2$, so that we must have $k \geq 3$. Let $Y=\mathbb{P}^{2} \times$ $\mathbb{P}^{2} \times\left(\mathbb{P}^{1}\right)^{k-2}$ and fix $o \in \mathbb{P}^{2} \backslash \pi_{2}\left(Y^{\prime}\right)$. Set $M:=\pi_{2}^{-1}(o)$, and $\Lambda:=\langle\nu(M)\rangle$. Then $r=9 \cdot 2^{k-2}-1, \operatorname{dim} \Lambda=9 \cdot 2^{k-3}-1$. Let $r^{\prime}:=9 \cdot 2^{k-3}-1$ and consider the linear projection $\ell: \mathbb{P}^{r} \backslash \Lambda \rightarrow \mathbb{P}^{r^{\prime}}$ from $\Lambda$ which acts on $\nu(Y)$ as the composition of the Segre embedding and the map $\mathbb{P}^{2} \times$ $\mathbb{P}^{2} \times\left(\mathbb{P}^{1}\right)^{k-2} \backslash \mathbb{P}^{2} \times\{o\} \times\left(\mathbb{P}^{1}\right)^{k-2} \rightarrow \mathbb{P}^{2} \times\left(\mathbb{P}^{1}\right)^{k-1}$, which is the linear projection $\mathbb{P}^{2} \backslash\{o\} \rightarrow \mathbb{P}^{1}$ on the second factor and the identity on any other factor. Since $\left(Y^{\prime} \cup\{p\}\right) \cap M=\emptyset, \ell(q)$ is well defined. We conclude since we already proved the statement in the case where only one of the factors is a $\mathbb{P}^{2}$.

\section{Lemmas}

In this section, we collect the basic lemmas that we will need all along the proof of the main theorem of the present paper, Theorem 7.1.

The following two lemmas describe two very basic properties that two different sets $A$ and $B$ evincing the rank of the same rank-3 point $q$ have to satisfy.

Lemma 4.1. Let $q$ be a not-identifiable tensor and let $A$ and $B$ two distinct sets evincing the rank of $q$. Define $S:=A \cup B$. If $\sharp(S) \geq 5$ and $\operatorname{dim}\langle\nu(S)\rangle=2$, then the rank of $q$ cannot be 3 .

Proof. Assume the existence of such a rank-3 tensor $q$ with 2 distinct decompositions $A$ and $B$ s.t. $\sharp(A \cup B) \geq 5$. The plane $\langle\nu(S)\rangle$ contains at least five not-collinear points. Note that $\langle\nu(S)\rangle \nsubseteq X$, otherwise also $q \in X$ which contradicts $r_{X}(q)=3$. So $\langle\nu(S)\rangle \cap X$ contains a conic $\mathcal{C}$. Either if it is reduced or not, the two secant variety of $\mathcal{C}$ fills $\langle\nu(S)\rangle=\mathbb{P}^{2}$. So $r_{X}(q) \leq 2$, which is an absurd.

Lemma 4.2. Let $q$ be a not-identifiable rank-3 tensor and let $A, B \in \mathcal{S}(Y, q)$ be distinct. Then $\sharp(A \cap B) \leq 1$.

Proof. Suppose, by contradiction, that $A$ and $B$ have 2 distinct points in common and call the set of these two points $E$. Let $A=E \cup\{u\}$ and $B=$ $E \cup\{v\}$. Since the rank of $q$ is $3, q \notin\langle\nu(E)\rangle$, but since by definition $q \in$ $\langle\nu(A)\rangle \cap\langle\nu(B)\rangle$ we have that $\langle\nu(E)\rangle \subsetneq\langle\nu(A)\rangle \cap\langle\nu(B)\rangle$. Clearly $\langle\nu(E)\rangle$ is a line; therefore, $\operatorname{dim}\langle\nu(A)\rangle \cap\langle\nu(B)\rangle>1$, but $\langle\nu(A)\rangle$ and $\langle\nu(B)\rangle$ are both planes, so $\langle\nu(A)\rangle=\langle\nu(B)\rangle$. In the plane $\langle\nu(A)\rangle$, we have two different lines: $\nu(E)$ and $\langle\nu(u), \nu(v)\rangle$, which mutually intersect in at most a point $q^{\prime}$. Remark that $q^{\prime} \notin X$ because otherwise the line $\langle\nu(E)\rangle$ would have at least 3 points of rank 1 and so we would have $\langle\nu(E)\rangle \subset X$, contradicting Remark 1.10. So $r_{X}\left(q^{\prime}\right)=2$ and $\sharp \mathcal{S}\left(Y, q^{\prime}\right) \geq 2$, by Proposition 2.1 we get that actually $q^{\prime} \in\left\langle\nu\left(Y^{\prime}\right)\right\rangle$, where $Y^{\prime}=\mathbb{P}^{1} \times \mathbb{P}^{1}$. But also $E,\{u, v\} \subset Y^{\prime}$, so $q \in\left\langle\nu\left(Y^{\prime}\right)\right\rangle$, which contradicts the fact that $q$ has rank 3 .

An immediate corollary of Lemma 4.2 is the following. 
Corollary 4.3. If $q$ is a rank-3 tensor and $A$ and $B$ are two distinct sets evincing its rank, then the cardinality of $A \cup B$ can only be either 5 or 6 .

This corollary turns out to be extremely useful for the proof of our main result, Theorem 7.1. We will be allowed to focus only on the structure of notidentifiable points of rank-3 with at least two decompositions $A$ and $B$ as in Corollary 4.3. This is the reason why we will study separately the case $\sharp A \cup B=5$ in Sect. 5 from the case $\sharp A \cup B=6$ in Sect. 6 .

Another very useful behaviour that needs to be understood to study the identifiability of rank-3 tensors, is the structure of the not-independent sets of at most 3 rank-1 tensors. This is what is described by the following lemma.

Lemma 4.4. A set of points $E \subset Y \simeq \mathbb{P}^{n_{1}} \times \cdots \times \mathbb{P}^{n_{k}}$ of cardinality at most 3 does not impose independent conditions to multilinear forms over $Y_{i} \simeq \mathbb{P}^{n_{1}} \times \cdots \times \hat{\mathbb{P}}_{i} \times \cdots \times \mathbb{P}^{n_{k}}, i=1, \ldots, k$ (i.e. $\left.h^{1}\left(\mathcal{I}_{E}\left(\hat{\varepsilon}_{i}\right)\right)>0\right)$ if and only if one of the following cases occurs:

1. $\sharp(E)=3$ and there is $j \in\{1, \ldots, k\} \backslash\{i\}$ such that $\sharp\left(\pi_{h}(E)\right)=1$ for all $h \notin\{i, j\}$;

2. there are $u, v \in E$ such that $u \neq v$ and $\eta_{i}(u)=\eta_{i}(v)$.

Proof. The fact that both items 1. and 2. imply that $h^{1}\left(\mathcal{I}_{E}\left(\hat{\varepsilon}_{i}\right)\right)>0$ is obvious. Let us describe the other implication.

By definition $H^{0}\left(\mathcal{O}_{Y}\left(\hat{\varepsilon}_{i}\right)\right) \cong H^{0}\left(\mathcal{O}_{Y_{i}}(1, \ldots, 1)\right.$, and $\mathcal{O}_{Y}\left(\hat{\varepsilon}_{i}\right)$ is not a very ample line bundle. So we cannot be sure about the injectivity of the restriction $\eta_{i \mid E}$ of $\eta_{i}$ to the finite set $E$.

If $\eta_{i \mid E}$ is not injective one immediately gets that $h^{1}\left(\mathcal{I}_{E}\left(\hat{\varepsilon}_{i}\right)\right)>0$. Moreover, if $\eta_{i \mid E}$ is not injective it means that there are 2 distinct points of $E$, say $u$ and $v$ which are mapped by $\eta_{i}$ onto the same point, i.e. we are in item 2 . of this lemma.

Now assume that $\eta_{i \mid E}$ is injective (i.e. we are not in item 2.). This implies that $\sharp E=\sharp \eta_{i}(E)$. We have by hypothesis that $h^{1}\left(\mathcal{I}_{E}\left(\hat{\varepsilon}_{i}\right)\right)>0$. Since by definition $h^{1}\left(\mathcal{I}_{E}\left(\hat{\varepsilon}_{i}\right)\right)=h^{1}\left(Y_{i}, \mathcal{I}_{\eta_{i}(E)}(1, \ldots, 1)\right)$ we have that $\eta_{i}(E)$ does not impose independent conditions to the multilinear forms over $Y_{i}$; therefore, $\sharp\left(\eta_{i}(E)\right) \geq 3$ which clearly implies that $\sharp\left(\eta_{i}(E)\right)=3$ since by hypothesis the cardinality of $E$ is at most 3 . Now $\eta_{i}(E)$ is a set of 3 distinct points on $Y_{i}$ which does not impose independent conditions to the multilinear forms over $Y_{i}$, and $\mathcal{O}_{Y_{i}}(1, \ldots, 1)$ is very ample, therefore the 3 points of $\eta_{i}(E)$ must be mapped to collinear points by the Segre embedding $\nu_{i}$ of $Y_{i}$. Hence, by the structure of the Segre variety $\nu_{i}\left(Y_{i}\right)$, we get that $\left\langle\nu_{i}\left(\eta_{i}(E)\right)\right\rangle \subseteq \nu_{i}\left(Y_{i}\right)$ and there is $j \in\{1, \ldots, k\} \backslash\{i\}$ such that $\sharp\left(\pi_{h}\left(\eta_{i}(E)\right)\right)=1$ for all $h \notin\{i, j\}$. Since $h \neq i$, we have $\pi_{h}\left(\eta_{i}(E)\right)=\pi_{h}(E)$.

\section{Two Different Solutions with One Common Point}

We have seen in Corollary 4.3 that if a rank-3 tensor $q$ is not-identifiable and $A, B$ are two sets of points on the Segre variety computing its rank, then 
$\sharp A \cup B$ can only be either 5 or 6 . This section is fully devoted to the case in which $\sharp A \cup B=5$, i.e. $A$ and $B$ share only one point and call it $p$ :

$$
S:=A \cup B, \sharp S=5, \quad A \cap B=\{p\} \text { and } A^{\prime}=A \backslash\{p\}, \quad B^{\prime}=B \backslash\{p\} .
$$

The matrix case is well known, therefore we will always assume that $q$ is an order- $k \geq 3$ tensor, i.e. $q \in\langle\nu(Y)\rangle$ with $Y=\prod_{i=1}^{k} \mathbb{P}^{n_{i}}$ and $k \geq 3$.

We will study separately the cases in which:

- $Y$ contains at least one factor of projective dimension 2 and all the others of dimension either 1 or 2 (Proposition 5.1);

- $Y$ is a product of $\mathbb{P}^{1} \mathrm{~s}$ only (see Proposition 5.2).

This will completely cover the cases of not-identifiable rank-3 tensors with the condition (5.1) since, by Remark 1.9, the concise Segre of a rank-3 point $q$ is $X_{q}=\nu\left(\mathbb{P}^{n_{1}} \times \cdots \times \mathbb{P}^{n_{k}}\right)$, with $n_{1}, \ldots, n_{k} \in\{1,2\}$.

Proposition 5.1. Let $Y$ be the multiprojective space with at least 3 factors and at least one them of projective dimension 2, i.e. $Y=\mathbb{P}^{2} \times \mathbb{P}^{n_{2}} \times \cdots \times \mathbb{P}^{n_{k}}$ with $n_{i} \in\{1,2\}$ for $i=1, \ldots, k$ and $k \geq 3$. Let $q \in \sigma_{3}^{0}(\nu(Y))$, with $\nu(Y)$ the concise Segre of $q$. If there exist two sets $A, B \in \mathcal{S}(Y, q)$ evincing the rank of $q$ such that $\sharp A \cap B=1$ then $q$ is as in Proposition 3.10 .

Proof. Consider a divisor $M \in\left|\mathcal{O}_{Y}\left(\varepsilon_{1}\right)\right|$ containing $A^{\prime}=A \backslash\{p\}$. By Concision/Autharky $S \nsubseteq M$, so, by Lemma 1.13, either $h^{1}\left(\mathcal{I}_{S \backslash S \cap M}\left(\hat{\varepsilon}_{1}\right)\right)>0$ or $p \notin M$ and $A^{\prime} \cup B^{\prime} \subset M$. We study separately the two cases.

1. First assume $h^{1}\left(\mathcal{I}_{S \backslash S \cap M}\left(\hat{\varepsilon}_{1}\right)\right)>0$.

The divisor $M$ contains $A^{\prime}$ by definition so $\sharp(S \backslash S \cap M) \leq 3$; moreover, if we define $Y_{1}:=\mathbb{P}^{n_{2}} \times \cdots \times \mathbb{P}^{n_{k}}$ with $n_{i}=1,2$ for $i=2, \ldots, k$, we have that $\mathcal{O}_{Y_{1}}(1, \ldots, 1)$ is very ample; therefore, we can apply Lemma 4.4 and say that one of the following occurs:

(i) $\sharp(S \backslash S \cap M)=3$ and there exists a projection $\pi_{i}$, with $i \in\{2, \ldots, k\}$ such that $\sharp\left(\pi_{i}(S \backslash S \cap M)\right)=1$;

(ii) There exist $u, v \in(S \backslash S \cap M)$ such that $u \neq v$ and $\eta_{1}(u)=\eta_{1}(v)$. We remark that case (ii) implies that $\pi_{i}(u)=\pi_{i}(v)$ for all $i>1$. Since $M$ contains $A^{\prime}$, we have that $S \backslash S \cap M=\{u, v\} \subseteq B$, we can exclude case (ii) thanks to Remark 1.10 .

So only case (i) is possible. Since $\sharp(S \backslash S \cap M)=3$ we have that $S \backslash S \cap$ $M=B$ and there exist an index $i \in\{2, \ldots, k\}$ such that $\sharp \pi_{i}(S \backslash S \cap M)=$ 1. The fact that there is $i \in\{2, \ldots, k\}$ such that $\sharp\left(\pi_{i}(B)\right)=1$, means that $B$ only depends by $k-1$ factors, contradicting Autarky.

2. Now assume $A^{\prime} \cup B^{\prime} \subset M$.

Let $Y^{\prime \prime}$ be the minimal multiprojective space contained in $M$ and containing $A^{\prime} \cup B^{\prime}$. Since $\left.q \in\left\langle\left\langle\nu\left(Y^{\prime \prime}\right)\right\rangle \cup\{p\}\right)\right\rangle$ and $p \notin Y^{\prime \prime}$, there is a unique $o \in\left\langle\nu\left(Y^{\prime \prime}\right)\right\rangle$ such that $q \in\langle\{\nu(p), o\}\rangle$. Since $\langle\nu(A)\rangle(\operatorname{resp} .\langle\nu(B)\rangle)$ is a plane containing $\nu(p)$ and $q$, there is a unique $o_{1} \in\left\langle\nu\left(A^{\prime}\right)\right\rangle$ (resp. $o_{2} \in\left\langle\nu\left(B^{\prime}\right)\right\rangle$ ) such that $q \in\left\langle\left\{\nu(p), o_{1}\right\}\right\rangle$ (resp. $q \in\left\langle\left\{\nu(p), o_{2}\right\}\right\rangle$ ). The uniqueness of $o$ gives $o=o_{1}=o_{2}$. Since $o_{1}=o_{2}$, we get a tensor of rank 2 with $A^{\prime}$ and $B^{\prime}$ as solutions. Thus, $q$ is as described in Proposition 3.10 . 
Proposition 5.2. Let $Y=\left(\mathbb{P}^{1}\right)^{\times k}$ with $k \geq 3$ and let $q \in \sigma_{3}^{0}(\nu(Y))$ be such that there exist two different sets $A, B \in \mathcal{S}(Y, q)$ with the property $\sharp(A \cup B)=5$, where $\nu(Y)$ is the concise Segre of $q$. Then $k$ can only be either 3 or 4 . If $k=3$ then $q$ belongs to a tangent space of $\nu\left(\left(\mathbb{P}^{1}\right)^{\times 3}\right)$ and $\operatorname{dim}(\mathcal{S}(Y, q)) \geq 2$. If $k=4$ then $\operatorname{dim}(\mathcal{S}(Y, q)) \geq 1$.

Proof. If $k=3$ then the only rank-3 tensors in $\left.\left\langle\nu\left(\mathbb{P}^{1}\right)^{\times 3}\right)\right\rangle$ are those belonging to the the tangential variety of the Segre variety (cf. [9,24]) for which $\operatorname{dim}(\mathcal{S}(Y, q)) \geq 2($ cf. $[1,8,27,28])$.

The case $k=4$ is covered by Remark 3.2.

Assume $k>4$ and write $Y=\prod_{i=1}^{k} \mathbb{P}_{i}^{1}$. Let $S=A \cup B$ as in (5.1).

We build a recursive set of divisors to being able to cover the whole set $S$ as follows. Let $o_{i} \in \mathbb{P}_{i}^{1}, i=2,3,4$ be such that:

1st divisor $\pi_{4}^{-1}\left(o_{4}\right) \cap S \neq \emptyset$ and call $M_{4}:=\pi_{4}^{-1}\left(o_{4}\right)$;

2nd divisor $\pi_{3}^{-1}\left(o_{3}\right) \cap\left(S \backslash\left(S \cap M_{4}\right)\right) \neq \emptyset$ and call $M_{3}:=\pi_{3}^{-1}\left(o_{3}\right)$.

3rd divisor If $M_{3} \cup M_{4}$ already covers the whole $S$ (i.e. $S \subset M_{3} \cup M_{4}$ ), set $M_{2}$ to be any divisor $M_{2} \in\left|\mathcal{O}_{Y}\left(\hat{\varepsilon}_{2}\right)\right|$.

4th divisor Otherwise, if $S \nsubseteq M_{3} \cup M_{4}$, choose $o_{2} \in \mathbb{P}_{2}^{1}$ such that $\pi_{2}^{-1}\left(o_{2}\right) \cap$ $\left(S \backslash S \cap\left(M_{3} \cup M_{4}\right)\right) \neq \emptyset$ and set $M_{2}:=\pi_{2}^{-1}\left(o_{2}\right)$.

Now it may happen that either $S \subset M_{2} \cup M_{3} \cup M_{4}$ or not. We study those two cases in (a) and (b), respectively.

(a) Here we assume that $S \subset M_{2} \cup M_{3} \cup M_{4}$. Since $\sharp(S)=5$ there is at least one of the $M_{i}$ s containing at least two points of $S$, and there are two of the $M_{i}$ s whose union contains at least 4 points of $S$ : wlog we may assume that $\sharp\left(S \cap\left(M_{3} \cup M_{4}\right)\right) \geq 4$.

- Assume $\sharp\left(S \cap\left(M_{3} \cup M_{4}\right)\right)=4$. Since $\mathcal{O}_{Y}(1,1,0,0, \ldots)$ is globally generated, we have that $h^{1}\left(\mathcal{I}_{S \backslash S \cap\left(M_{3} \cup M_{4}\right)}(1,1,0,0,1,1, \ldots)\right)=0$, contradicting Lemma 1.13.

- Assume $S \subset M_{3} \cup M_{4}$. Therefore, there is one of the $M_{i}$ s containing at least 3 points of $S$, let $\sharp\left(M_{4} \cap S\right) \geq 3$. Since $S \nsubseteq M_{4}$, we get $h^{1}\left(\mathcal{I}_{S \backslash S \cap M_{4}}\left(\hat{\varepsilon}_{4}\right)\right)>0$ (by Lemma 1.13); hence, $\sharp\left(S \backslash S \cap M_{4}\right)=2$ and

$$
S \backslash S \cap M_{4}=\{u, v\} \text { with } \pi_{i}(u)=\pi_{i}(v), \forall i \neq 4 .
$$

Since $h^{1}\left(\mathcal{I}_{S \backslash S \cap M_{3}}\left(\hat{\varepsilon}_{3}\right)\right)>0$ (again by Lemma 1.13, we get that either there are $w, z \in S \backslash S \cap M_{3}$ such that $w \neq z, \pi_{i}(w)=\pi_{i}(z)$ for all $i \neq 3$ or $\nu_{4}\left(\eta_{4}\left(S \cap M_{4}\right)\right.$ ) (remind Notation 1.3) is made by 3 collinear points, say with a line corresponding to the $i$ th factor. The latter case cannot arise because $S$ does not depend only on the third, fourth and $i$ th factor of $Y$. Thus, there exist

$w, z \in S \backslash S \cap M_{3}$ such that $w \neq z, \pi_{i}(w)=\pi_{i}(z) \forall i \neq 3$.

In (5.2) and (5.3), we have 4 distinct points $u, v, w, z$ such that $\sharp\left(\pi_{5}(\{u, v, w, z\})\right)=1$. Take $M_{5} \in\left|\mathcal{O}_{Y}\left(\varepsilon_{5}\right)\right|$ containing $\{u, v, w, z\}$. Since $h^{1}\left(\mathcal{I}_{S \backslash S \cap M_{5}}\left(\hat{\varepsilon}_{5}\right)\right)=0$, Autarky and Lemma 1.13 give a contradiction. 
(b) Assume $S \nsubseteq M_{2} \cup M_{3} \cup M_{4}$. By Lemma 1.13, we get $h^{1}\left(\mathcal{I}_{S \backslash S \cap\left(M_{2} \cup M_{3} \cup M_{4}\right)}\right.$ $(1,0,0,0,1,1, \ldots))>0$. Thus, $\sharp\left(S \backslash\left(M_{2} \cup M_{3} \cup M_{4}\right)\right)=2$, say $S \backslash\left(M_{2} \cup\right.$ $\left.M_{3} \cup M_{4}\right)=\{u, v\}$ and $\pi_{i}(u)=\pi_{i}(v)$ for all $i \neq 2,3,4$. But in this case it is sufficient to change the original choice of $o_{4}$ and take as $O_{4}$ the point $\pi_{4}(u)$ and the the new divisor $M_{4}$ will contain 2 points of $S$, i.e. $u, v$ therefore we are able to get new divisors $M_{2}, M_{3}$ with the same construction as above leading to the case $S \subset M_{2} \cup M_{3} \cup M_{4}$ excluded in step (a).

\section{Two Disjoint Solutions}

We have seen in Corollary 4.3 that if a rank-3 tensor $q$ is not-identifiable and $A, B$ are two sets of points on the Segre variety computing its rank, then $\sharp A \cup B$ can only be either 5 or 6 . This section is fully devoted to the case in which $\sharp A \cup B=6$, i.e. $A$ and $B$ are disjoint:

$$
S:=A \cup B, \sharp S=6, \quad A:=\left\{a_{1}, a_{2}, a_{3}\right\}, B:=\left\{b_{1}, b_{2}, b_{3}\right\} \quad A \cap B=\emptyset .
$$

First of all, let us show that if $q$ is a rank-3 tensor whose concise Segre $\nu(Y)$ has at least two factors of projective dimension 2 , it never happens that in $\mathcal{S}(Y, q)$ there are two disjoint sets.

Remark 6.1. Let $Y=\left(\mathbb{P}^{2}\right)^{\times k_{1}} \times\left(\mathbb{P}^{1}\right)^{\times k_{2}}$ and $S \subset Y$ a set of 6 distinct points. Consider $I \subseteq\left\{k_{1}+1, \ldots, k_{1}+k_{2}\right\}$ and $\varepsilon:=\sum_{i \in I} \varepsilon_{i}$. Suppose there exists a divisor $M \in\left|\mathcal{O}_{Y}(\varepsilon)\right|$ intersecting $S$ in 4 points. Call $\{u, v\}:=S \backslash(S \cap M)$. In this setting one can apply Lemma 1.13 and get that $h^{1}\left(\mathcal{I}_{\{u, v\}}(\widehat{\varepsilon})\right)>0$ (where $\widehat{\varepsilon}$ is a $\left(k_{1}+k_{2}\right)$-tuple with $0 \mathrm{~s}$ in position of the indices appearing in $\varepsilon$ of $I$ and 1 s everywhere else) and $\pi_{h}(u)=\pi_{h}(v)$ for any $h \in\left\{1, \ldots, k_{1}+k_{2}\right\} \backslash I$.

Proposition 6.2. Let $Y$ be a multiprojective space with at least three factors and at least two of them of projective dimension 2, i.e. $Y=\mathbb{P}^{2} \times \mathbb{P}^{2} \times \mathbb{P}^{n_{3}} \times$ $\cdots \times \mathbb{P}^{n_{k}}$ with $n_{i} \in\{1,2\}$ for $i=1, \ldots, k$ and $k \geq 3$. Let $q \in \sigma_{3}^{0}(\nu(Y))$, with $\nu(Y)$ the concise Segre of $q$. If $A, B \in \mathcal{S}(Y, q)$ evince the rank of $q$, then $A$ and $B$ cannot be disjoint.

Proof. The proof is by absurd: assume that there exist $A, B \in \mathcal{S}(Y, q)$ with $A \cap B=\emptyset$. By Remark 3.3, we have that $\left\langle\pi_{i}(A)\right\rangle=\left\langle\pi_{i}(B)\right\rangle=\mathbb{P}^{2}$ for $i=1,2$. Fix $W \in\left|\mathcal{I}_{B}\left(\varepsilon_{2}+\varepsilon_{3}\right)\right|$ (it exists, because $h^{0}\left(\mathcal{O}_{Y}\left(\varepsilon_{2}+\varepsilon_{3}\right)\right)=$ $\left.h^{0}\left(\mathbb{P}^{2} \times \mathbb{P}^{n_{3}}, \mathcal{O}_{\mathbb{P}^{2} \times \mathbb{P}^{n_{3}}}(1,1)\right)=3\left(n_{3}+1\right)>4\right)$. Since $\pi_{1 \mid A}$ is injective, we have $h^{1}\left(\mathcal{I}_{A}\left(\varepsilon_{1}\right)\right)=0$. Thus, $S \subset W$ by Lemma 1.13. In this way, we have shown that

$$
\text { any divisor } D \in\left|\mathcal{O}_{Y}\left(\varepsilon_{2}+\varepsilon_{3}\right)\right| \text { containing } B \text { contains also } A \text {. }
$$

Claim 6.2.1 $\pi_{3}\left(a_{i}\right)=\pi_{3}\left(b_{i}\right)$ where $a_{i}, b_{i}$ are as in (6.1), for $i=1,2,3$.

The proof of this claim can be repeated verbatim for all the other projections with only one caution that we will highlight in the sequel. Therefore, by repeating the argument for all the projections, we will get that $\pi_{j}\left(a_{i}\right)=\pi_{j}\left(b_{i}\right)$ for $i=1,2,3$ and for $j=1, \ldots, k$ which is a contradiction with $A$ and $B$ being distinct. This will conclude the proof. 
Proof of the Claim 6.2.1. Take a general hyperplane $J_{3} \subset \mathbb{P}^{n_{3}}$ containing $\pi_{3}\left(b_{i}\right)$, (where the $b_{i}$ s are as in $\left.(6.1), i=1,2,3\right)$ by genericity we may assume that if $n_{3}=2$ then $J_{3}$ is a line which does not contain any other point of that projection. Set $M_{3}:=\pi_{3}^{-1}\left(J_{3}\right)$. Take a line

$L_{2} \subset \mathbb{P}^{2}$ containing $\left\{\pi_{2}\left(b_{j}\right), \pi_{2}\left(b_{k}\right)\right\}$ with $j, k \neq i$ and set $M_{2}:=\pi_{2}^{-1}\left(L_{2}\right)$.

We have $B \subset M_{2} \cup M_{3} \in\left|\mathcal{O}_{Y}\left(\varepsilon_{2}+\varepsilon_{3}\right)\right|$. Thus, from (*), we get that $M_{2} \cup M_{3}$ contains also $A$. Since $A \nsubseteq M_{2}$ by Autarky, there is $a \in A \cap M_{3}$, i.e. there is $a \in A$ such that

$$
\pi_{3}(a)=\pi_{3}\left(b_{i}\right)
$$

(in fact if $n_{3}=1$ it is trivial, if $n_{3}=2$ then we have already remarked that $\pi_{3}\left(b_{i}\right)$ is the only point of $J_{3}$ belonging to $\left.\pi_{3}(Y)\right)$. Since $\pi_{i \mid A}$ is injective for $i=1,2$ (cf. Remark 3.3), the points of $A$ projecting on $\pi_{3}\left(b_{i}\right)$ are different for different $i$ s except if there are $b_{i} \neq b_{j}$ such that $\pi_{3}\left(b_{i}\right)=\pi_{3}\left(b_{j}\right)$. Suppose that this is the case. By Lemma 4.4 we get that $\sharp\left(S \backslash S \cap M_{3}\right)=2$. Thus, if for $i \neq j \pi_{3}\left(b_{i}\right)=\pi_{3}\left(b_{j}\right)$ there are 2 points of $A$ and 2 points of $B$ in $M_{3}$, i.e. $\sharp\left(S \cap M_{3}\right)=4$. Suppose that $S \cap M_{3}=\left\{a_{3}, b_{3}, a_{2}, b_{2}\right\}$. By [13, Lemmas 2.4 and 2.5] (also [7, Lemma 5.1, item (b)]) $h^{1}\left(\mathcal{I}_{S \backslash S \cap M_{3}}\left(\hat{\varepsilon}_{3}\right)\right)>0$, i.e. $\pi_{i}\left(a_{1}\right)=\pi_{i}\left(b_{1}\right)$ for all $i \neq 3$. This is a contradiction since we already know that $\pi_{3}\left(a_{2}\right)=\pi_{3}\left(b_{2}\right)$ and we would have $a_{2}=b_{2}$, which contradicts the assumption that $A \cap B=\emptyset$.

Therefore, the points $a \in A$ of (6.2) are all different for different choices of $i$ s. So we may assume that $\pi_{3}\left(a_{i}\right)=\pi_{3}\left(b_{i}\right)$ for $i=1,2,3$ and the $\pi_{3}\left(b_{i}\right) \neq$ $\pi_{3}\left(b_{j}\right)$ for $i \neq j$.

The argument of the proof of Claim 6.2.1 can be repeated verbatim for all the others $\pi_{j}$ s with the only caution that when we do the case $j=2$ we have to use a line $L_{1} \subset \mathbb{P}^{2}$ containing $\left\{\pi_{1}\left(b_{j}\right), \pi_{1}\left(b_{k}\right)\right\}$ with $j, k \neq i$ and set $M_{1}:=\pi_{1}^{-1}\left(L_{1}\right)$ instead of $M_{2}$ and $L_{2}$ in $\left(^{* *}\right)$. Moreover, $\left({ }^{*}\right)$ clearly holds if we replace the $\varepsilon_{2}$ with $\varepsilon_{1}$ and $\varepsilon_{3}$ with $\varepsilon_{j}$ for any $j=3, \ldots, k$. As already highlighted this concludes the proves since $\pi_{j}\left(a_{i}\right)=\pi_{j}\left(b_{i}\right)$ for $i=1,2,3$ and for $j=1, \ldots, k$ which is a contradiction with $A$ and $B$ being distinct.

This shows that under the assumption (6.1), we can exclude the case where the Segre variety has at least two factors of projective dimension 2 .

Let us focus on the four-factor case.

Proposition 6.3. Let $Y=\mathbb{P}^{2} \times \mathbb{P}^{1} \times \mathbb{P}^{1} \times \mathbb{P}^{1}$. Let $q \in \sigma_{3}^{0}(\nu(Y))$, with $\nu(Y)$ the concise Segre of $q$. There do not exist two disjoint sets $A, B \in \mathcal{S}(Y, q)$ evincing the rank of $q$.

Proof. Assume by contradiction that there exist two disjoint sets $A, B \in$ $\mathcal{S}(Y, q)$ evincing the rank of $q$ and, moreover, assume that no $\eta_{i \mid S}$ is injective, for $i=2,3,4$.

By Remark 1.10, for each $i=2,3,4$ there exists $a \in A, b \in B$ such that $\eta_{i}(a)=\eta_{i}(b)$. Fix $H:=\pi_{1}^{-1}(L)$, where $L \subset \mathbb{P}^{2}$ is a line containing $\pi_{1}\left(a_{1}\right)$ and $\pi_{1}\left(a_{2}\right)$, where $a_{1}, a_{2} \in A$. Since we assumed that no $\eta_{i \mid S}$ is injective, then there exist $b_{1}, b_{2} \in B$ such that $\pi_{1}\left(a_{i}\right)=\pi_{1}\left(b_{i}\right)$, for $i=1,2$. Thus, $H \supset\left\{a_{1}, a_{2}, b_{1}, b_{2}\right\}$ and by Autarky $S \not \subset H$, so there is at least an element of $S$ 
out of $H$, e.g. $a_{3} \in S \backslash\left\{a_{1}, a_{2}, b_{1}, b_{2}\right\}$. Thus, we have $h^{1}\left(\mathcal{I}_{S \backslash S \cap H}(0,1,1,1)\right)=0$ contradicting Lemma 1.13. So there exists at least one integer $h \in\{2, \ldots, 4\}$ such that $\eta_{h \mid S}$ is injective.

First, define recursively the integers such that the preimages of points $o \in \mathbb{P}^{1}$ intersect maximally the set $S$ :

$$
\alpha_{4}:=\max \left\{\sharp\left(\pi_{i}^{-1}(o) \cap S\right)\right\}_{o \in \mathbb{P}^{1} ; i=2, \ldots, 4} .
$$

By rearranging if necessary, we can assume that the index $i=2, \ldots, 4$ realizing $\alpha_{4}$, is $i=4$. Call $K_{4}:=\pi_{4}^{-1}(o)$. Then define

$$
\alpha_{3}:=\max \left\{\sharp\left(\pi_{i}^{-1}(o) \cap\left(S \backslash\left(S \cap K_{4}\right)\right)\right)\right\}_{o \in \mathbb{P}^{1} ; i=2,3} .
$$

By rearranging if necessary, we can assume that the index $i=2,3$ realizing $\alpha_{3}$, is $i=3$. Call $K_{3}:=\pi_{3}^{-1}(o)$. Finally define

$$
\alpha_{2}:=\max \left\{\sharp\left(\pi_{2}^{-1}(o) \cap\left(S \backslash\left(S \cap K_{4} \cup K_{3}\right)\right)\right)\right\}_{o \in \mathbb{P}^{1}} .
$$

So if we denote by $o_{j} \in \mathbb{P}^{1}, j=2,3,4$ the points realizing $\alpha_{2}, \alpha_{3}, \alpha_{4}$, respectively, then we call

$$
K_{j}:=\pi_{j}^{-1}\left(o_{j}\right) \text { for } j=2,3 .
$$

Remark that by Autarky assumption $1 \leq \alpha_{3} \leq \alpha_{4} \leq 5$.

It is easy to see that $\alpha_{4}$ cannot be 5 . In fact if $\alpha_{4}=5$, then $\sharp\left(S \backslash S \cap K_{4}\right)=1$ which implies that $h^{1}\left(\mathcal{I}_{S \backslash S \cap K_{4}}(1,1,1,0)\right)=0$, which is a contradiction with Lemma 1.13.

So the possibilities for $\alpha_{3}$ and $\alpha_{4}$ are $1 \leq \alpha_{3} \leq \alpha_{4} \leq 4$.

Let us show that

$$
\alpha_{2} \neq 1
$$

Assume that $\left(\alpha_{2}, \alpha_{3}, \alpha_{4}\right)=(1,1,1)$. In such a case, the divisor $K_{2} \cup$ $K_{3} \cup K_{4} \in\left|\mathcal{O}_{Y}\left(\hat{\varepsilon}_{1}\right)\right|$ would contain exactly 3 points of $S$. Moreover, if $h^{1}$ $\left(\mathcal{I}_{S \backslash\left(S \cap K_{2} \cup K_{3} \cup K_{4}\right)}\left(\varepsilon_{1}\right)\right)>0$ then by Lemma 4.4 we would have a contradiction with $\left(\alpha_{3}, \alpha_{4}\right)=(1,1)$. Therefore, if $\left(\alpha_{2}, \alpha_{3}, \alpha_{4}\right)=(1,1,1)$, we must have $h^{1}\left(\mathcal{I}_{S \backslash\left(S \cap K_{2} \cup K_{3} \cup K_{4}\right)}\left(\varepsilon_{1}\right)\right)=0$, but this is a contradiction with Lemma 1.13. Thus, if $\alpha_{2}=1$ then $K_{3} \cup K_{4}$ should contain at least 3 points of $S$, i.e. $\alpha_{3} \geq 1$ and $\alpha_{4} \geq 2$.

Now assume that $\left(\alpha_{2}, \alpha_{3}\right)=(1,1)$. Then $\pi_{3 \mid S}$ is injective. The idea is to build a divisor $F \in\left|\mathcal{O}_{Y}(\varepsilon)\right|$ with $\varepsilon=\sum_{i \in I} \varepsilon_{i}$, for some finite $I \in\{1, \ldots, k\}$, such that $\sharp(S \backslash F \cap S)=2$ and apply Remark 6.1 to $F$ : the existence of such an $F$ will contradict the injectivity of $\pi_{3 \mid S}$. Let $H_{i} \in\left|\mathcal{O}_{Y}\left(\varepsilon_{i}\right)\right|$ such that $H_{i} \cap$ $\left(S \backslash S \cap K_{4}\right) \neq \emptyset$ for $i=2,3$. The divisor $F$ is either $F=K_{4}$, or $F=K_{4} \cup H_{3}$ or $K_{4} \cup H_{2} \cup H_{3}$ if $\alpha_{4}=4,3,2$, respectively. The case $\left(\alpha_{2}, \alpha_{3}, \alpha_{4}\right)=(1,2,2)$ can be easily excluded since $\sharp\left(S \backslash S \cap K_{2} \cup K_{3} \cup K_{4}\right)=1$ and by Lemma 1.13 , we would have $h^{1}\left(\mathcal{I}_{S \backslash S \cap\left(K_{2} \cup K_{3} \cup K_{4}\right)}\left(\varepsilon_{1}\right)\right)>0$, which is absurd. For the same reason, $\left(\alpha_{2}, \alpha_{3}, \alpha_{4}\right)=(1,2,3)$ is also impossible because then $\sharp\left(S \cap\left(K_{3} \cup\right.\right.$ $\left.\left.K_{4}\right)\right)=5$ and by Lemma 1.13 we would have $h^{1}\left(\mathcal{I}_{S \backslash S \cap\left(K_{3} \cup K_{4}\right)}(1,1,0,0)\right)>0$, which is a contradiction. This shows $\alpha_{2} \neq 1$.

We are, therefore, left with $\alpha_{2} \neq 1<\alpha_{3} \leq \alpha_{4}=2,3,4$. 
Suppose that $\alpha_{3}=\alpha_{4}=2$. With these assumption one also gets $\alpha_{2}=2$. Indeed on the one hand, we just showed that we may always take $H \in$ $\left|\mathcal{O}_{Y}\left(\varepsilon_{2}\right)\right|$ such that $\left.\sharp\left(S \backslash S \cap\left(K_{3} \cup K_{4}\right)\right) \cap H\right) \neq 0$, so such a $H$ intersects $S$ non-trivially and $K_{2}$ is among those $H$ s. On the other hand, $\alpha_{2} \neq 1$ by $(\dagger)$. So $\sharp\left(S \cap K_{2}\right)=2$. By the construction of the $K_{i}$ s in $(6.6)$ for $i=2,3$, 4 , it is easy to show that

$$
S=\coprod_{i=2}^{4} S \cap K_{i}
$$

So, since $S=\coprod_{i=2}^{4} S \cap K_{i}$ and $\sharp\left(S \cap K_{i}\right)=2$ for $i=2,3$, 4, we can apply Remark 6.1 separately to the divisors $K_{i} \cup K_{j}$ with $i \neq j$ and get that $h^{1}\left(\mathcal{I}_{S \cap K_{i}}\left(\varepsilon_{1}+\varepsilon_{i}\right)\right)>0$ for $i=2,3,4$ and so $\pi_{1}\left(S \cap K_{i}\right)=1$ for $i=2,3,4$. To get a contradiction it is sufficient to apply again Remark 6.1 to $\pi_{1}^{-1}\left(\left\langle\pi_{1}(S \cap\right.\right.$ $\left.\left.\left.K_{3}\right), \pi_{1}\left(S \cap K_{2}\right)\right\rangle\right)$. This shows that $\sharp\left(\pi_{i}\left(S \cap K_{4}\right)\right)=1$ for $i=2,3$, 4 . Now since also $\sharp\left(\pi_{1}\left(S \cap K_{4}\right)\right)=1$, then $\sharp\left(S \cap K_{4}\right)=1$, which is a contradiction with the assumption $\alpha_{3}=2$.

This proves that $1<\alpha_{2} \leq \alpha_{3}$, and $2<\alpha_{4}=3,4$.

The case $\left(\alpha_{3}, \alpha_{4}\right)=(2,4)$ can be excluded using the same argument of the case $\left(\alpha_{2}, \alpha_{3}, \alpha_{4}\right)=(2,2,2)$ above applying Remark 6.1 since if $\left(\alpha_{3}, \alpha_{4}\right)=$ $(2,4)$ we have that $K_{4}$ plays the role of $M$ in the remark.

We are, therefore, left with the unique possibility of $\left(\alpha_{3}, \alpha_{4}\right)=$ $(3,3)$.

Claim 6.3.1 $\sharp\left(\pi_{2}\left(S \cap K_{4}\right)\right)=1$.

Proof of Claim 6.3.1:. Since we are in the hypothesis $\alpha_{4}=3$, the projection of $S \cap K_{4}$ onto the first two factors of $Y$ is made by at most 3 points.

Suppose that such a projection is made by exactly 3 points. Call $Z$ the image of the projection of $S \cap K_{4}$ onto the first two factors. Since $h^{1}\left(\mathbb{P}^{2} \times\right.$ $\left.\mathbb{P}^{1}, \mathcal{I}_{Z}(1,1)\right)>0$ those points must lie on a line $L$ when applying the Segre embedding. Moreover, from Remark 3.3 we know that $\pi_{1}(A)$ and $\pi_{1}(B)$ are sets of linearly independent points and since linear subspaces of the Segre variety are all contained in a factor, we get that $L \subset \mathbb{P}^{2}$. Thus, $\sharp\left(\pi_{2}\left(S \cap K_{4}\right)\right)=$ 1 proving the claim in this case.

If the projection of $S \cap K_{4}$ onto the first two factors is made by less than 3 points, there exist at least two points, $u, v \in S \cap K_{4}$ such that they share the same image under the projection. Remark that if we consider $E \subset S \cap K_{4}$ such that $\sharp E=2$ and take $T \in\left|\mathcal{I}_{E}(1,1,0,0)\right|$, then $T \supset S \cap K_{4}$. Indeed if $S \cap K_{4} \not \subset T$ then we have that $T \cup K_{3}$ contains exactly five points of $S$, which leads to a contradiction because by Lemma 1.13 we would have $h^{1}\left(\mathcal{I}_{S \backslash S \cap T \cup K_{3}}\left(\hat{\varepsilon}_{3}\right)\right)>0$. Therefore, also the third point of $S \cap K_{4}$ share the same image of $u$ and $v$ and we are done.

Using the third factor instead of the second one, one gets $\sharp\left(\pi_{3}\left(K_{4} \cap S\right)\right)=$ 1 and since we assumed that $\alpha_{4}$ is reached on the fourth factor we also have $\sharp\left(\pi_{4}\left(K_{4} \cap S\right)\right)=1$. The same argument can be applied to $S \cap K_{3}$ which leads to $\sharp\left(\pi_{2}\left(K_{3} \cap S\right)\right)=\sharp\left(\pi_{4}\left(K_{3} \cap S\right)\right)=1$. Thus, $\sharp\left(\pi_{i}\left(K_{4} \cap S\right)\right)=\sharp\left(\pi_{i}\left(K_{3} \cap S\right)\right)=1$ for all $i>1$ which contradicts Autarky. 
Since the identifiability of rank-3 tensors in $\left\langle\nu\left(\left(\mathbb{P}^{1}\right)^{\times 4}\right)\right\rangle$ is already fully described by Remark 3.2, we are therefore done with the order- 4 tensors and we can focus on tensors of order bigger or equal than 5. So we will deal with $Y=\mathbb{P}^{n_{1}} \times\left(\mathbb{P}^{1}\right)^{l}$, with $n_{1}=1,2$ and $l \geq 4$.

Lemma 6.4. Let $q$ be a rank-3 tensor of order at least 5 and let $\nu(Y)$ be its concise Segre. If there exist two disjoint sets $A, B \in \mathcal{S}(Y, q)$ as in (6.1), then there exists at least an index $i \in\{1, \ldots, k\}$ such that $\eta_{i \mid S}$ and $\pi_{i \mid S}$ are injective.

Proof. [Injectivity of $\eta_{i \mid S}$.]

Assume that no $\eta_{i \mid S}$ is injective, then by Remark 1.10 for any $i=$ $1, \ldots, k$ there exist an element $a \in A$ and an element $b \in B$ such that $\pi_{h}(a)=\pi_{h}(b)$ for any $h \neq i$. It is easy to check that this condition, applied to two disjoint sets of 3 points each, and at least five $\eta_{i} \mathrm{~s}$, imposes either that $A \cap B \neq \emptyset$ (contradiction) or that one of the two sets (either $A$ or $B$ ) depends only on 4 factors (contradicting Autarky).

[Injectivity of $\pi_{i \mid S}$.]

Assume that $\eta_{i \mid S}$ is injective and that $\pi_{i \mid S}$ is not injective. If $i=1$ and the first factor of $Y$ is $\mathbb{P}^{2}$ take $H \in\left|\mathcal{O}_{Y}\left(\varepsilon_{i}\right)\right|$ as the preimage of a general line that contains exactly one point of $S$; otherwise take $H \in\left|\mathcal{O}_{Y}\left(\varepsilon_{i}\right)\right|$ as the preimage of a point of $S$. We remark that in both cases we get that $\sharp\left(\pi_{i}(S \cap H)\right)=1$. Since by Autarky $S \not \subset H$, by Lemma 1.13, we have that

$$
h^{1}\left(\mathcal{I}_{S \backslash S \cap H}\left(\hat{\varepsilon}_{i}\right)\right)>0 .
$$

We distinguish different cases depending on $\sharp(S \backslash S \cap H)$.

1. Assume $\sharp(S \backslash S \cap H)=4$ and call $S^{\prime}:=\eta_{i}(S \backslash S \cap H)$; let $A^{\prime} \subset S^{\prime}$ such that $\sharp A^{\prime}=2$ and call $B^{\prime}:=S^{\prime} \backslash A^{\prime}$, so $\sharp B^{\prime}=2$. Since $\eta_{i \mid S}$ is injective we have that $h^{1}\left(Y_{i}, \mathcal{I}_{S^{\prime}}\left(\hat{\varepsilon_{i}}\right)\right)=h^{1}\left(\mathcal{I}_{S \backslash S \cap H}\left(\hat{\varepsilon}_{i}\right)\right)>0$. So $\left\langle\nu_{i}\left(A^{\prime}\right)\right\rangle \cap\left\langle\nu_{i}\left(B^{\prime}\right)\right\rangle \neq \emptyset$, which means that we have at least a point $q^{\prime} \in\left\langle\nu_{5}\left(Y_{i}\right)\right\rangle$ of rank 2 for which $A^{\prime}$ and $B^{\prime}$ are different subsets evincing its rank. Thus, by Proposition 2.3, since $\sharp \mathcal{S}\left(Y_{i}, q^{\prime}\right)>1$, the points in $A^{\prime}$ and $B^{\prime}$ only depend on two factors, i.e. $\sharp\left(\pi_{j}\left(S^{\prime}\right)\right)=1$ for at least two indices $j \in\{1, \ldots, k\}$. Without loss of generality assume it happens for $j=1,2$. If the first factor of $Y$ is $\mathbb{P}^{2}$, let $M_{1} \in\left|\mathcal{I}_{\pi_{1}\left(S^{\prime}\right)}\left(\varepsilon_{1}\right)\right|$ be the preimage of a general line containing $\pi_{1}\left(S^{\prime}\right)$ and let $\left\{M_{2}\right\}:=\left|\mathcal{I}_{\pi_{2}\left(S^{\prime}\right)}\left(\varepsilon_{2}\right)\right|$. Otherwise let $\left\{M_{j}\right\}:=$ $\left|\mathcal{I}_{\pi_{j}\left(S^{\prime}\right)}\left(\varepsilon_{j}\right)\right|$, for $j=1,2$; in both cases then $h^{1}\left(\mathcal{I}_{S \backslash S \cap M_{j}}\left(\hat{\varepsilon}_{j}\right)\right)>0$. So $S \backslash S \cap M_{j}=S \cap H$ and $\sharp\left(\eta_{j}(S \cap H)\right)=1$, for $j=1,2$. If we call $S \cap H=\{u, v\}$, it follows that $\eta_{1}(u)=\eta_{1}(v)$ and $\eta_{2}(u)=\eta_{2}(v)$, so in particular we get that $\pi_{j}(u)=\pi_{j}(v)$ for any $j$, which is a contradiction.

2. Assume $\sharp(S \backslash S \cap H)=3$. By Proposition 4 .4 there exists $j \neq i$ such that $\sharp\left(\pi_{h}(S \backslash S \cap H)\right)=1$ for all $h \neq i, j$. For all $h>1$ with $h \neq j, i$, since $h^{0}\left(\mathcal{O}_{Y}\left(\varepsilon_{h}\right)\right)=2$ we get $h^{0}\left(\mathcal{I}_{S \backslash S \cap H}\left(\varepsilon_{h}\right)\right)=1$. Set $\left\{M_{h}\right\}:=\left|\mathcal{I}_{S \backslash S \cap H}\left(\varepsilon_{h}\right)\right|$, if $h=1$ and the first factor is $\mathbb{P}^{2}$ take $M_{h} \in\left|\mathcal{I}_{S \backslash S \cap H}\left(\varepsilon_{h}\right)\right|$ as the preimage of a general line, otherwise call $\left\{M_{h}\right\}:=\left|\mathcal{I}_{S \backslash S \cap H}\left(\varepsilon_{h}\right)\right|$. Since we took $H$ such that $\sharp \pi_{i}(S \cap H)=1$, there exists at least an index $t \neq i$ such that $\sharp \pi_{t}(S \cap H) \geq 2$. Thus, we can find $D \in\left|\mathcal{O}_{Y}\left(\varepsilon_{t}\right)\right|$ containing exactly one point of $S \cap H$. 
For all $s \neq t$, set $W_{s}:=M_{s} \cup D$, so $\sharp\left(S \backslash S \cap W_{s}\right)=2$; we remark that $W_{j} \cap S=W_{s} \cap S$ for any $j, s$ thus we may call $E:=S \backslash S \cap W_{s}$.

By Lemma 1.13, we have that $h^{1}\left(\mathcal{I}_{E}\left((1, \ldots, 1)-\varepsilon_{s}-\varepsilon_{t}\right)\right)>0$, so $\sharp \pi_{j}(E)=1$ for all $j \neq s, t$. Since $E \subset H$, we have that $\pi_{i}(E)=1$; moreover, taking $s=1,2,3$, if $t \neq j$, we get that $\sharp E=1$; thus, a contradiction. It remains to study what happens when $t=j$, i.e. if $\sharp\left(\pi_{j}(S \cap H)\right) \geq 2$. In such a case, when we let $s$ varies in $\{1, \ldots, k\} \backslash\{i, j\}$, we get $\sharp \pi_{s}(S \cap H)=1$. Thus, $\eta_{j}(S \cap H)=1$, i.e. the three points of $S \cap H$ actually lies on a line, which is a contradiction with Remark 1.10, because two of them are points of $A$ or $B$.

3. Assume $\sharp(S \backslash S \cap H) \leq 2$. Since $h^{1}\left(\mathcal{I}_{S \backslash S \cap H}\left(\hat{\varepsilon}_{i}\right)\right)>0$, we get that $\sharp(S \backslash S \cap$ $H)=2$ and that $\sharp \eta_{i}(S \backslash S \cap H)=1$, which is a contradiction.

With these two lemmas we can conclude the case of two disjoint sets $A, B \in \mathcal{S}(Y, q)$ with $q$ of rank-3.

Proposition 6.5. Let $q \in \sigma_{3}^{0}(\nu(Y))$ be a tensor of order $k \geq 5$ and let $\nu(Y)$ be its concise Segre. Then $\mathcal{S}(Y, q)$ does not contain two disjoint sets.

Proof. By Lemma 6.4 there exists at least an index $i \in\{1, \ldots, k\}$ such that $\eta_{i \mid S}$ is injective, from which follows that the corresponding $\pi_{i \mid S}$ is also injective. Now if $\eta_{j \mid S}$ is not injective for some $j \neq i$ then $\pi_{i \mid S}$ is not injective, which is a contradiction with the assumption that $\eta_{i \mid S}$ is injective. Therefore, thus $\eta_{j \mid S}$ and $\pi_{j \mid S}$ have to be injective for all $j=1, \ldots, k$.

Write $A:=\left\{a_{1}, a_{2}, a_{3}\right\}$ and $B:=\left\{a_{4}, a_{5}, a_{6}\right\}$. If the first factor is a $\mathbb{P}^{2}$ take $L_{1} \in \mathbb{P}^{2}$ as a general line containing $\pi_{1}\left(a_{1}\right)$ and define $H_{1} \in\left|\mathcal{I}_{a_{1}}\left(\varepsilon_{1}\right)\right|$ as $H_{1}:=\pi_{1}^{-1} L_{1}$. For $i=2, \ldots, 5$ take $\left\{H_{i}\right\}:=\left|\mathcal{I}_{a_{i}}\left(\varepsilon_{i}\right)\right|$ (this is possible since by hypothesis $k \geq 5$ ). Otherwise, for all $i=1, \ldots, k$ take $\left\{H_{i}\right\}:=$ $\left|\mathcal{I}_{a_{i}}\left(\varepsilon_{i}\right)\right|$. In both cases, since every $\pi_{i \mid S}$ is injective we get that $H_{1} \cup \cdots \cup$ $H_{5}$ contains exactly 5 points of $S$. Thus, from Lemma 1.13 , we get that $h^{1}\left(\mathcal{I}_{S \backslash\left(S \cap H_{1} \cup \ldots \cup H_{5}\right)}(0,0,0,0,0,1, \ldots, 1)\right)>0$ which is a contradiction since $\sharp\left(S \backslash\left(S \cap H_{1} \cup \cdots \cup H_{5}\right)\right)=1$.

\section{Identifiability of Rank-3 Tensors}

The following theorem completely characterizes the identifiability of any rank-3 tensor and it is the main theorem of the present paper.

Theorem 7.1. Let $Y=\mathbb{P}^{n_{1}} \times \cdots \times \mathbb{P}^{n_{k}}$ be the multiprojective space of the concise Segre of a rank-3 tensor $q$. Denote with $\mathcal{S}(Y, q)$, the set of all subsets of $Y$ computing the rank of $q$. The rank-3 tensor $q$ is identifiable except in the following cases:

1. $q$ is a rank-3 matrix, in this case $\operatorname{dim}(\mathcal{S}(Y, q))=6$;

2. $q$ belongs to a tangent space of the Segre embedding of $Y=\mathbb{P}^{1} \times \mathbb{P}^{1} \times \mathbb{P}^{1}$, in this case $\operatorname{dim}(\mathcal{S}(Y, q)) \geq 2$;

3. $q$ is an order- 4 tensor of $\sigma_{3}^{0}(Y)$ with $Y=\mathbb{P}^{1} \times \mathbb{P}^{1} \times \mathbb{P}^{1} \times \mathbb{P}^{1}$, in this case $\operatorname{dim}(\mathcal{S}(Y, q)) \geq 1$

4. $q$ is as in Example 3.6, where $Y=\mathbb{P}^{2} \times \mathbb{P}^{1} \times \mathbb{P}^{1}$, in this case $\operatorname{dim}(\mathcal{S}(Y, q))=$ 3 ; 
5. $q$ is as in Example 3.7, where $Y=\mathbb{P}^{2} \times \mathbb{P}^{1} \times \mathbb{P}^{1}$, in this case $\mathcal{S}(Y, q)$ contains two different four-dimensional families;

6. $q$ is as in Proposition 3.10, where $Y=\mathbb{P}^{n_{1}} \times \cdots \times \mathbb{P}^{n_{k}}$ is such that $k \geq 3, n_{i} \in\{1,2\}$ for $i=1,2, n_{i}=1$ for $i>2$ and $\sum_{i=1}^{k} n_{i} \geq 4$. In this case, $\operatorname{dim}(\mathcal{S}(Y, q)) \geq 2$ and if $n_{1}+n_{2}+k \geq 6$ then $\operatorname{dim}(\mathcal{S}(Y, q))=2$.

Proof. In case 1 . the point $q$ is a rank-3 matrix; therefore, it is highly notidentifiable. See Remark 3.1 for the computation of the dimension of $\mathcal{S}(Y, q)$.

Case 2. is also well known: see [8, Remark 3].

Case 3. corresponds to the defective 3rd secant variety of the Segre embedding of $Y=\left(\mathbb{P}^{1}\right)^{\times 4}$ and the fact that all the elements of $\sigma_{3}^{0}(\nu(Y))$ are not-identifiable is shown in $\operatorname{Remark} 3.2$. The fact that $\operatorname{dim}(\mathcal{S}(Y, q))=1$ for the generic rank-3 tensor depends on the fact that the 3rd defect $\delta_{3}$ of $\nu\left(\left(\mathbb{P}^{1}\right)^{\times 4}\right)$ is exactly 1 (cf. [1]). Moreover, by [43, Cap II, Ex 3.22, part (b)], we get that for any rank 3 tensor $q$, the $\operatorname{dimension} \operatorname{dim}(\mathcal{S}(Y, q)) \geq 1$.

Cases 4., 5. and 6. are treated in Examples 3.6 and 3.7 and in Proposition 3.10 , respectively.

All the above considerations prove that the list of cases enumerated in the statement corresponds to non-identifiable rank-3 tensors. We need to show that such a list is exhaustive. Since the matrix case is already fully covered by case 1 , we only need to care about tensors of order at least 3 .

First of all recall that by Remark 1.9, the concise Segre of a rank-3 tensor $q$ is $\nu\left(\mathbb{P}^{n_{1}} \times \cdots \times \mathbb{P}^{n_{k}}\right)$, with $n_{1}, \ldots, n_{k} \in\{1,2\}$. Then consider two distinct sets $A, B \in \mathcal{S}(Y, q)$. By Corollary 4.3, it can only happen that $\sharp(A \cup B)=5,6$.

If $\sharp(A \cup B)=5$, the fact that our list of not-identifiable rank-3 tensors is exhaustive is proved in Propositions 5.1 and 5.2.

If $\sharp(A \cup B)=6$, we can first use Proposition 6.2 to exclude the all the cases in which $Y$ has at least two factors of dimension 2. Then we start arguing by the number of factors of $Y$.

If $Y$ has 3 factors and it is the product of $\mathbb{P}^{1} \mathrm{~s}$ only, then the unique tensors of rank-3 are those of the tangential variety to the Segre variety and this is case 2 of our theorem. The case of $Y=\mathbb{P}^{2} \times \mathbb{P}^{1} \times \mathbb{P}^{1}$ is completely covered by Proposition 3.8 together with Examples 3.6 and 3.7 (cf. Corollary $3.9)$.

If $Y$ has 4 factors and one of them is a $\mathbb{P}^{2}$, there is Proposition 6.3 assuring that $\mathcal{S}(Y, q)$ does not contain two disjoint sets. If $Y$ is a product of four $\mathbb{P}^{1} \mathrm{~s}$, we are in case 3 of our theorem.

The fact that if $Y$ has at least 5 factors then $\mathcal{S}(Y, q)$ does not contain two disjoint sets is done in Proposition 6.5.

\section{Acknowledgements}

The first two authors were partially supported by GNSAGA of INDAM. We thank the referee for the useful comments.

Funding Open access funding provided by Università degli Studi di Trento within the CRUI-CARE Agreement. 
Open Access. This article is licensed under a Creative Commons Attribution 4.0 International License, which permits use, sharing, adaptation, distribution and reproduction in any medium or format, as long as you give appropriate credit to the original author(s) and the source, provide a link to the Creative Commons licence, and indicate if changes were made. The images or other third party material in this article are included in the article's Creative Commons licence, unless indicated otherwise in a credit line to the material. If material is not included in the article's Creative Commons licence and your intended use is not permitted by statutory regulation or exceeds the permitted use, you will need to obtain permission directly from the copyright holder. To view a copy of this licence, visit http:// creativecommons.org/licenses/by/4.0/.

Publisher's Note Springer Nature remains neutral with regard to jurisdictional claims in published maps and institutional affiliations.

\section{References}

[1] Abo, H., Ottaviani, G., Peterson, C.: Induction for secant varieties of Segre varieties. Trans. Am. Math. Soc. 361, 767-792 (2006)

[2] Ådlandsvik, B.: Joins and higher secant varieties. Math. Scand. 61, 213-222 (1987)

[3] Allman, E.S., Rhodes, J.A.: The identifiability of tree topology for phylogenetic models, including covarion and mixture models. J. Comput. Biol. 13, 1101-1113 (2006)

[4] Angelini, E., Chiantini, L., Mazzon, A.: Identifiability for a class of symmetric tensors. Mediterr. J. Math. 16, 97 (2019)

[5] Angelini, E., Chiantini, L., Vannieuwenhoven, N.: Identifiability beyond Kruskal's bound for symmetric tensors of degree 4. Atti Accad. Naz. Lincei Rend. Lincei Mat. Appl. 29, 465-485 (2018)

[6] Ballico, E., Bernardi, A.: A uniqueness result on the decompositions of a bihomogeneous polynomial. Linear Multilinear Algebra. 65, 677-698 (2017)

[7] Ballico, E., Bernardi, A.: Stratification of the fourth secant variety of Veronese variety via the symmetric rank. Adv. Pure Appl. Math. 4, 215-250 (2013)

[8] Ballico, E., Bernardi, A.: Tensor ranks on tangent developable of Segre varieties. Linear Multilinear Algebra. 61, 881-894 (2013)

[9] Ballico, E., Bernardi, A.: On the ranks of the third secant variety of SegreVeronese embeddings. Linear Multilinear Algebra. 67, 583-597 (2019)

[10] Ballico, E., Bernardi, A., Catalisano, M.V.: Higher secant varieties of $\mathbb{P}^{n} \times \mathbb{P}^{1}$ embedded in bi-degree $(a, b)$. Comm. Algebra 40, 3822-3840 (2012)

[11] Ballico, E., Bernardi, A., Catalisano, M.V., Chiantini, L.: Grassmann secants, identifiability, and linear systems of tensors. Linear Algebra Appl. 438, 121-135 (2013)

[12] Ballico, E., Bernardi, A., Chiantini, L.: On the dimension of contact loci and the identifiability of tensors. Ark. Mat. 56, 265-283 (2018)

[13] Ballico, E., Bernardi, A., Christandl, M., Gesmundo, F.: On the partially symmetric rank of tensor product of $\mathrm{W}$-states and other symmetric tensors. Rend. Lincei Math. Appl. 30, 93-124 (2019) 
[14] Bates, D.J., Hauenstein, J.D., Sommese, A.J., Wampler, C.W.: Bertini: Software for Numerical Algebraic Geometry. (2006). www.bertini.nd.edu

[15] Baur, K., Draisma, J.: Secant dimensions of low-dimensional homogeneous varieties. Adv. Geom. 10, 1-29 (2010)

[16] Bernardi, A.: Ideals of varieties parameterized by certain symmetric tensors. J. Pure Appl. Algebra 212, 1542-1559 (2008)

[17] Bernardi, A., Carlini, E., Catalisano, M.V.: Higher secant varieties of $\mathbb{P}^{n} \times \mathbb{P}^{m}$ embedded in bi-degree $(1, d)$. J. Pure Appl. Algebra 215, 2853-2858 (2011)

[18] Bernardi, A., Carlini, E., Catalisano, M.V., Gimigliano, A., Oneto, A.: The Hitchhiker guide to: Secant varieties and tensor decomposition. Mathematics 6, 314 (2018)

[19] Bernardi, A., Carusotto, I.: Algebraic Geometry tools for the study of entanglement: an application to spin squeezed states. J. Phys. A: Math. Theor. $\mathbf{4 5}$ (2012)

[20] Bernardi, A., Daleo, N.S., Hauenstein, J.D., Mourrain, B.: Tensor decomposition and homotopy continuation. Differ. Geom. Appl. 55, 78-105 (2017)

[21] Bernardi, A., Vanzo, D.: A new class of non-identifiable skew-symmetric tensors. Ann. di Mat. Pura ed Appl. 197, 1499-1510 (2018)

[22] Bocci, C., Chiantini, L.: On the identifiability of binary Segre products. J. Algebraic Geom. 22, 1-11 (2011)

[23] Bocci, C., Chiantini, L., Ottaviani, G.: Refined methods for the identifiability of tensors. Ann. di Mat. Pura ed Appl. 193, 1691-1702 (2014)

[24] Buczyński, J., Landsberg, J.M.: On the third secant variety. J. Algebraic Comb. 40, 475-502 (2014)

[25] P. Bürgisser, M. Clausen and M.A. Shokrollahi. Algebraic complexity theory. Grundlehren der mathematischen Wissenschaften. (1997)

[26] Casarotti, A., Mella, M.: From non Defectivity to Identifiability. arXiv:1911.00780

[27] Catalisano, M.V., Geramita, A.V., Gimigliano, A.: Ranks of tensors, secant varieties of Segre varieties and fat points. Linear Algebra Appl. 355, 263-285 (2002). Erratum, Linear Algebra Appl. 367, 347-348 (2003)

[28] Catalisano, M.V., Geramita, A.V., Gimigliano, A.: Higher secant varieties of the Segre-Veronese varieties. In: projective varieties with unexpected properties. Walter de Gruyter, Berlin, pp. 81-107 (2005)

[29] Catalisano, M.V., Geramita, A.V., Gimigliano, A.: Higher secant varieties of the Segre varieties $\mathbb{P}^{1} \times \cdots \times \mathbb{P}^{1}$. J. Pure Appl. Algebra 201, 367-380 (2005)

[30] Chiantini, L.: Hilbert Function and Tensor Analysis. In: E. Ballico, A. Bernardi, I. Carusotto, S. Mazzucchi, V. Moretti (Eds.), Quantum Physics and Geometry, Lecture Notes of the Unione Matematica Italiana 25, pp. 125-151 (2019)

[31] Chiantini, L., Ciliberto, C.: Weakly defective varieties. Trans. Am. Math. Soc. 354, 151-178 (2001)

[32] Chiantini, L., Ciliberto, C.: On the concept of $k$-secant order of a variety. J. Lond. Math. Soc. 2, 436-454 (2006)

[33] Chiantini, L., Ciliberto, C.: The Grassmannians of secant varieties of curves are not defective. Indag. Math. (N.S.), 13, 23-28 (2002)

[34] Chiantini, L., Ottaviani, G.: On generic identifiability of 3-tensors of small rank. SIAM J. Matrix Anal. Appl. 33, 1018-1037 (2011) 
[35] Chiantini, L., Ottaviani, G., Vannieuwenhoven, N.: An algorithm for generic and low-rank specific identifiability of complex tensors. SIAM J. Matrix Anal. Appl. 35, 1265-1287 (2014)

[36] Chiantini, L., Ottaviani, G., Vannieuwenhoven, N.: Effective Criteria for specific identifiability. SIAM J. Matrix Anal. Appl. 38, 656-681 (2017)

[37] Ciliberto, C., Mella, M., Russo, F.: Varieties with one apparent double point. J. Algebraic Geometry. 13, 475-512 (2004)

[38] Domanov, I., De. Lathauwer, L.: On the uniqueness of the canonical polyadic decomposition of third-order tensors-part II: Uniqueness of the overall decomposition. SIAM J. Matrix Anal. Appl. 34, 876-903 (2013)

[39] Domanov, I., De. Lathauwer, L.: Canonical polyadic decomposition of thirdorder tensors: reduction to generalized eigenvalue decomposition. SIAM J. Matrix Anal. Appl. 35, 636-660 (2014)

[40] Domanov, I., De. Lathauwer, L.: Canonical polyadic decomposition of thirdorder tensors: relaxed uniqueness conditions and algebraic algorithm. Linear Algebra Appl. 513, 342-375 (2017)

[41] Domanov, I., De. Lathauwer, L.: Generic uniqueness conditions for the canonical polyadic decomposition and IND-SCAL. SIAM J. Matrix Anal. Appl. 36, 1567-1589 (2015)

[42] Galuppi, F., Mella, M.: Identifiability of homogeneous polynomials and Cremona transformations. J. Reine Angew. Math. 757, 279-308 (2019)

[43] Hartshorne, R.: Algebraic Geometry. Springer-Verlag, New York (1977)

[44] Holweck, F., Jaffali, H., Nounouh, I.: Grovers algorithm and the secant varieties. Quantum Inf. Process. 15, 4391-4413 (2016)

[45] Kruskal, B.: Three-way arrays: Rank and uniqueness of trilinear decompositions, with application to arithmetic complexity and statistics. Linear Algebra Appl. 18, 95-138 (1977)

[46] Jiang, T., Sidiropoulos, N.D.: Kruskal's permutation lemma and the identification of CANDECOMP/PARAFAC and bilinear models with constant modulus constraints. IEEE Trans. Signal Process. 52, 2625-2636 (2004)

[47] Laface, A., Postinghel, E.: Secant varieties of Segre-Veronese embeddings of $\left(\mathbb{P}^{1}\right)^{r}$. Math. Ann. 356, 1455-1470 (2013)

[48] Landsberg, J.M.: Tensors: Geometry and Applications. Graduate Studies in Mathematics. Amer. Math. Soc. Providence, 128 (2012)

[49] Landsberg, J.M.: Geometry and Complexity Theory. Cambridge Studies in Advanced Mathematics. 169 (2017)

[50] Massarenti, A., Mella, M., Staglianò, G.: Effective identifiability criteria for tensors and polynomials. J. Symb. Comput. 87, 227-237 (2018)

[51] Orús, R.: A practical introduction to tensor networks: matrix product states and projected entangled pair states. Ann. Phys. 349, 117-158 (2014)

[52] Rao, W., Li, D., Zhang, J.Q.: A tensor-based approach to L-shaped arrays processing with enhanced degrees of freedom. IEEE Signal Process. Lett. 25, $1-5(2018)$ 
Edoardo Ballico, Alessandra Bernardi and Pierpaola Santarsiero

Dipartimento di Matematica

University of Trento

Trento

Italy

e-mail: p.santarsiero-10unitn.it

Edoardo Ballico

e-mail: edoardo.ballico@unitn.it

Alessandra Bernardi

e-mail: alessandra.bernardi@unitn.it

Received: February 5, 2020.

Revised: January 30, 2021.

Accepted: May 24, 2021. 